\title{
Non-equilibrium Scaling Properties of a Double Quantum Dot System: Comparison between Perturbative Renormalization Group and Flow Equation Approach
}

\author{
V. Koerting, $, 1,2,3$, 田 P. Fritsch, ${ }^{4}$ and S. Kehrein ${ }^{4}$ \\ ${ }^{1}$ Department of Physics, University of Basel, Klingelbergstrasse 82, CH-4056 Basel, Switzerland \\ ${ }^{2}$ Niels Bohr Institute, Universitetsparken, DK-2100 Copenhagen Ø, Denmark \\ ${ }^{3}$ The Niels Bohr International Academy, The Niels Bohr Institute, \\ Blegdamsvej 17, DK-2100 Copenhagen, Denmark \\ ${ }^{4}$ Physics Department, Arnold Sommerfeld Center for Theoretical Physics and Center for NanoScience, \\ Ludwig-Maximilians-Universität, Theresienstrasse 37, 80333 Munich, Germany
}

(Dated: December 5, 2018)

\begin{abstract}
Since the experimental realization of Kondo physics in quantum dots, its far-from-equilibrium properties have generated considerable theoretical interest. This is due to the interesting interplay of non-equilibrium physics and correlation effects in this model, which has by now been analyzed using several new theoretical methods that generalize renormalization techniques to non-equilibrium situations. While very good agreement between these methods has been found for the spin-1/2 Kondo model, it is desirable to have a better understanding of their applicability for more complicated impurity models. In this paper the differences and commons between two such approaches, namely the flow equation method out of equilibrium and the frequency-dependent poor man's scaling approach are presented for the non-equilibrium double quantum dot system. This will turn out to be a particularly suitable testing ground while being experimentally interesting in its own right. An outlook is given on the quantum critical behavior of the double quantum dot system and its accessibility with the two methods.
\end{abstract}

PACS numbers: $05.10 . \mathrm{Cc}, 73.63 . \mathrm{Kv}, 72.10 . \mathrm{Fk}, 75.30 . \mathrm{Hx}, 72.15 . \mathrm{Qm}$

Keywords: Kondo impurities, out of equilibrium scaling theories

\section{INTRODUCTION}

The recent advances in nanotechnology permit to probe far-from-equilibrium transport properties of correlated electron systems. The paradigm for such experiments is the realization of Kondo physics in Coulomb blockade quantum dots 1 . This combination of nonequilibrium with correlation effects is theoretically challenging and has led to considerable efforts to develop suitable theoretical tools. The key challenge is that beyond linear response theory one does in general not know how to systematically construct the steady current-carrying state, except by following a difficult real time evolution problem.

During the past five years new powerful methods, both numerical and analytical, have been developed and used for investigating non-equilibrium quantum many-body problems. As analytical renormalization group methods have played an enormous role in understanding correlation physics in equilibrium, a number of these methods are directly based on renormalization ideas and carry them over to non-equilibrium situations: the frequencydependent renormalization group 2,3 , the real time renormalization group ${ }^{4,5}$ and the flow equation method ${ }^{6-8}$. While these methods show very good agreement for the Kondo model, it is clearly desirable for future applications to understand their relation and respective advantages in more detail.

In this paper we address this question for the frequency-dependent renormalization group and the flow equation method in the case of a more complicated quan- tum impurity model, namely for a double-dot system in the Kondo regime. In this system two Kondo dots are coupled via a spin-spin interaction, which is a setup related to recent experiments 9,10 . In addition, this model is particularly interesting in the present context for two additional reasons: i) It is known to exhibit an interesting quantum phase transition in equilibrium ${ }^{11}$ and one expects non-equilibrium properties to be especially important at quantum phase transitions. ii) Based on the investigation of the non-equilibrium spin-1/2 Kondo model, one knows that the decoherence generated by the steady state current plays the key role in understanding the far-from-equilibrium properties ${ }^{2,12}$. Now decoherence enters via two seemingly very different mechanisms in the frequency-dependent renormalization group and the flow equation method. For the frequency-dependent renormalization group one identifies suitable Korringalike decoherence rates that are then used to explicitly cut off the renormalization flow ${ }^{2,13}$. On the other hand, in the flow equation approach decoherence terms appear as two-loop contributions in the scaling equations ${ }^{6,7}$. For the conventional spin-1/2 Kondo system the different renormalization-based approaches show very good quantitative agreement including line-shapes and Korringa rates $5,7,8$.

The difference how decoherence enters highlights the respective advantages of these approaches: the frequency-dependent renormalization group (like real time RG) has a straightforward diagrammatic representation, while the flow equation method treats both manyparticle coherence effects (like Kondo physics) and deco- 
herence on the same footing in the scaling equations. In the following we will analyze how these differences manifest themselves in the non-equilibrium double-dot system. Previous studies of the non-equilibrium double-dot system based on the frequency-dependent renormalization group and non-equilibrium perturbation theory have been published in Refs. 14, 15.

This paper is organized as follows. In section [I] we first introduce the Hamiltonian of the double quantum dot system and discuss the various approximations used in its derivation. Section III explains the flow equation method and shows its application to the double dot system. In section IV we then introduce the perturbative renormalization group approach and discuss the scaling equations for our system. After comparing the two methods in leading logarithmic order in section $\nabla$ we discuss generalizations of the two approaches in section VI which include decoherence effects. At the end of section VI we then compare the two approaches again and show that the results at the decoherence scale are in very good agreement, although the underlying methodologies are quite different.

\section{MODEL}

The simplest model to illustrate the competition between a spin singlet and a Kondo singlet formation is the two-impurity Kondo model. It has been studied in detail Ref. 11 and gained new life with the progress in nanotechnology and the possibility to study two single quantum dots interacting with each other ${ }^{9,10}, 16$.

Here we study the problem of two quantum dots where two artificial impurities are attached to leads, in contrast to the historical two-impurity model which contains two impurities embedded in a metal 11 .

The double quantum dot (DQD) model describes two spin- $1 / 2$ degrees of freedom denoted as $\vec{S}_{L}$ and $\vec{S}_{R}$, which are each Kondo coupled to conduction band electrons with an additional mutual spin exchange interaction $H_{\mathrm{ex}}=K \vec{S}_{L} \vec{S}_{R}$, which is assumed to be antiferromagnetic $K>0$. The Hamiltonian of the system is given by

$$
H=H_{\text {leads }}+H_{\text {ex }}+H_{\text {Kondo }}
$$

The conduction band electrons are described by

$$
H_{\text {leads }}=\sum_{j} \sum_{k, \sigma} \epsilon_{\mathrm{k}, \mathrm{j}}: c_{k j \sigma}^{\dagger} c_{k j \sigma},
$$

where the lead index $j$ is specified later on, $\epsilon_{\mathrm{k}, \mathrm{j}}$ is the energy of the corresponding conduction band electron and $c_{k j \sigma}^{\dagger}\left(c_{k j \sigma}\right)$ are the corresponding creation (annihilation) operators for a conduction electron with momentum $k$ and spin $\sigma$. The notation : ...: denotes normal ordering with respect to the non-interacting ground state.
The Kondo interaction with the leads is

$$
\begin{aligned}
H_{\text {Kondo }}= & \sum_{j} \sum_{k^{\prime} k} J_{k^{\prime} k}^{L j}: \vec{S}_{L} \vec{s}_{\left(k^{\prime} j\right)(k j)}: \\
& +\sum_{j} \sum_{k^{\prime} k} J_{k^{\prime} k}^{R j}: \vec{S}_{R} \vec{s}_{\left(k^{\prime} j\right)(k j)}: .
\end{aligned}
$$

where $J_{k^{\prime} k}^{L j}\left(J_{k^{\prime} k}^{R j}\right)$ is the coupling of the left (right) quantum dot spin to the spin density of the conduction band electrons in the lead $j$

$$
: \vec{s}_{\left(k^{\prime} j\right)(k j)}:=\sum_{\sigma^{\prime} \sigma} \frac{1}{2} \vec{\tau}_{\sigma \sigma^{\prime}}: c_{k^{\prime} j \sigma^{\prime}}^{\dagger} c_{k j \sigma}:
$$

and $\vec{\tau}$ are the Pauli matrices of a spin-1/2.

In the two-impurity model the spin-spin interaction between the Kondo spins is mediated by the RKKY interaction ${ }^{18}$, i.e. for antiferromagnetic coupling generated by two Kondo spin-flip interactions. The RKKY interaction depends in sign and strength on the distance between the two impurities and since it is an effective interaction in $J^{2}$ it is both retarded and small (at least every reasonable theory should do so). Note that the RKKY interaction in this case scales to the same degree as the Kondo interaction in scaling theory.

On the contrary singlet-triplet states in quantum dots can arise from other physical effects, for example from exchange couplings and/or orbital degeneracies. Therefore the effective spin-spin interaction between the Kondo impurities can be tuned independently from the Kondo interaction with the leads $\underline{9}$.

For both methods we therefore include the spin exchange interaction in the unperturbed Hamiltonian

$$
H_{0}=H_{\text {leads }}+H_{\text {ex }},
$$

and we treat the Kondo interaction $H_{\text {Kondo }}$ as a small perturbation. The eigenstates of the unperturbed double dot spin system are singlet $|0,0\rangle$ with a total spin $S=0$ and triplet states $|1, m\rangle$ with a total spin of $S=1$ where $m=\{-1,0,1\}$. The perturbative $R G$ focuses on the flow of a generalized coupling function and the scaling equation is derived by diagrammatic perturbation theory in the vertex. In order to do the perturbation theory a pseudo-particle representation for the spin operators is introduced. In the flow equation method the scaling equations are derived from infinitesimal unitary transformations. These involve mainly the commutation relations of operators and therefore the spin can be treated as an operator.

\section{A. Pseudoparticle representation}

In order to calculate diagrams in perturbation theory and also in perturbative RG, we introduce the pseudo particles $d_{\gamma}^{\dagger}$ which create a singlet or triplet state $\gamma \in$ 
$\left\{s, t_{-}, t_{0}, t_{+}\right\}$. The spin exchange interaction Hamiltonian is thus diagonal in the pseudo particle operators,

$$
H_{\mathrm{ex}}=-\frac{3}{4} K d_{s}^{\dagger} d_{s}+\frac{1}{4} K \sum_{\gamma} d_{t_{\gamma}}^{\dagger} d_{t_{\gamma}} .
$$

The left and right spin (upper and lower sign, respectively) can be represented by bond operators ${ }^{19}$ :

$$
\begin{array}{r}
S_{L / R}^{z}=\frac{1}{2}\left(d_{t_{+}}^{\dagger} d_{t_{+}}-d_{t_{-}}^{\dagger} d_{t_{-}} \pm d_{s}^{\dagger} d_{t_{0}} \pm d_{t_{0}}^{\dagger} d_{s}\right), \\
S_{L / R}^{+}=\left(S_{L / R}^{-}\right)^{\dagger}=\frac{1}{2}\left(d_{t_{0}}^{\dagger} d_{t_{-}}+d_{t_{+}}^{\dagger} d_{t_{0}} \pm d_{s}^{\dagger} d_{t_{-}} \mp d_{t_{+}}^{\dagger} d_{s}\right) .
\end{array}
$$

The constraint

$$
Q=d_{s}^{\dagger} d_{s}+d_{t_{+}}^{\dagger} d_{t_{+}}+d_{t_{0}}^{\dagger} d_{t_{0}}+d_{t_{-}}^{\dagger} d_{t_{-}}=1
$$

is fulfilled by calculating the physical observable from the expectation value

$$
\langle\mathcal{O}\rangle_{Q=1}=\lim _{\lambda \rightarrow \infty} \frac{\langle Q \mathcal{O}\rangle_{\lambda}}{\langle Q\rangle_{\lambda}},
$$

where $\langle\ldots\rangle_{\lambda}$ is the average over the Hamiltonian $H_{\lambda}=$ $H+\lambda Q$ where the constraint enters as a chemical potential which is set to infinity at the end of the calculation 20 .

It is convenient to introduce a matrix representation of the Kondo spins in the bond operator notation by defining a generalized Pauli matrix $\vec{T}_{\alpha}$ leading to

$$
\vec{S}_{\alpha}=\sum_{\gamma^{\prime} \gamma} \frac{1}{2} d_{\gamma^{\prime}}^{\dagger}\left(\vec{T}_{\alpha}\right)_{\gamma^{\prime} \gamma} d_{\gamma} .
$$

In the case of the exchange coupled double quantum dot system this generalized Pauli matrices are given by

$$
\begin{aligned}
& \vec{T}_{L}^{z}=\left(\begin{array}{cccc}
0 & 0 & 1 & 0 \\
0 & 1 & 0 & 0 \\
1 & 0 & 0 & 0 \\
0 & 0 & 0 & -1
\end{array}\right), \quad \vec{T}_{R}^{z}=\left(\begin{array}{cccc}
0 & 0 & -1 & 0 \\
0 & 1 & 0 & 0 \\
-1 & 0 & 0 & 0 \\
0 & 0 & 0 & -1
\end{array}\right) \\
& \vec{T}_{L}^{+}=\left(\vec{T}_{L}^{-}\right)^{\dagger}=\left(\begin{array}{cccc}
0 & 0 & 0 & 1 \\
-1 & 0 & 1 & 0 \\
0 & 0 & 0 & 1 \\
0 & 0 & 0 & 0
\end{array}\right) \\
& \vec{T}_{R}^{+}=\left(\vec{T}_{R}^{-}\right)^{\dagger}=\left(\begin{array}{cccc}
0 & 0 & 0 & -1 \\
1 & 0 & 1 & 0 \\
0 & 0 & 0 & 1 \\
0 & 0 & 0 & 0
\end{array}\right)
\end{aligned}
$$

Note that the lower right $3 \times 3$ matrix, i.e. the triplet states, represents the Pauli matrices for a spin-1.

Using this notation the interaction Hamiltonian (3) is given in the general form

$$
\begin{gathered}
H_{\text {int }}=\sum_{\alpha, j=L, R} \sum_{R} \sum_{\gamma^{\prime} \gamma} \frac{1}{k^{\prime} \sigma^{\prime} ; k \sigma} J_{\gamma^{\prime} \gamma}^{\alpha, j}\left(\vec{T}_{\alpha}\right)_{\gamma^{\prime} \gamma} \vec{\tau}_{\sigma^{\prime} \sigma} \\
\times d_{\gamma^{\prime}}^{\dagger} d_{\gamma}: c_{k^{\prime} j \sigma^{\prime}}^{\dagger} c_{k j \sigma}:
\end{gathered}
$$

During the renormalization we will find that the coupling between triplet states $J_{t t} d_{t_{m}}^{\dagger} d_{t_{m^{\prime}}}$ flows differently than for the spin couplings including a singlet-to-triplet transition $J_{s t} d_{t_{m}}^{\dagger} d_{s}$ and $J_{t s} d_{s}^{\dagger} d_{t_{m}}$.

\section{B. Spin notation}

If we do not introduce pseudoparticle states, but keep the spin operator as a quantity, we find that transitions between the eigenstates of the DQD are given by

$$
\begin{array}{r}
\left(\vec{S}_{L}+\vec{S}_{R}\right)|S, m\rangle \rightarrow|S, m\rangle, \\
\left(\left(\vec{S}_{L}-\vec{S}_{R}\right)+2 i\left(\vec{S}_{L} \times \vec{S}_{R}\right)\right)|0,0\rangle \rightarrow|1, m\rangle, \\
\left(\left(\vec{S}_{L}-\vec{S}_{R}\right)-2 i\left(\vec{S}_{L} \times \vec{S}_{R}\right)\right)|1, m\rangle \rightarrow|0,0\rangle .
\end{array}
$$

Therefore we rewrite the Hamiltonian in Eq. (3) for the flow equation treatment by

$$
\begin{aligned}
& H_{\mathrm{int}}=\sum_{j=L, R} \sum_{k^{\prime} k} J_{k^{\prime} k}^{\mathrm{sum}, j}:\left(\vec{S}_{L}+\vec{S}_{R}\right) \vec{s}_{\left(k^{\prime} j\right)(k j)}: \\
& +\sum_{j=L, R} \sum_{k^{\prime} k} P_{k^{\prime} k}^{j}:\left(\left(\vec{S}_{L}-\vec{S}_{R}\right)+2 i\left(\vec{S}_{L} \times \vec{S}_{R}\right)\right) \vec{s}_{\left(k^{\prime} j\right)(k j)}: \\
& +\sum_{j=L, R} \sum_{k^{\prime} k} M_{k^{\prime} k}^{j}:\left(\left(\vec{S}_{L}-\vec{S}_{R}\right)-2 i\left(\vec{S}_{L} \times \vec{S}_{R}\right)\right) \vec{s}_{\left(k^{\prime} j\right)(k j)}:
\end{aligned}
$$

where the couplings are defined by

$$
\begin{aligned}
J_{k^{\prime} k}^{\mathrm{sum}, j} & =\frac{1}{2}\left(J_{k^{\prime} k}^{L, j}+J_{k^{\prime} k}^{R, j}\right), \\
P_{k^{\prime} k}^{j} & =\frac{1}{2}\left(\frac{1}{2}\left(J_{k^{\prime} k}^{L, j}-J_{k^{\prime} k}^{R, j}\right)+Q_{k^{\prime} k}^{j}\right), \\
M_{k^{\prime} k}^{j} & =\frac{1}{2}\left(\frac{1}{2}\left(J_{k^{\prime} k}^{L, j}-J_{k^{\prime} k}^{R, j}\right)-Q_{k^{\prime} k}^{j}\right),
\end{aligned}
$$

The interaction $Q_{k^{\prime} k}^{j}: 2 i\left(\vec{S}_{L} \times \vec{S}_{R}\right) \vec{s}_{\left(k^{\prime} j\right)(k j)}$ : is per se not present in the initial setup, but it turns out that the system of equations does not close if it is not included. This leads to the following initial conditions for a general flow parameter $B$

$$
\begin{aligned}
J_{k^{\prime} k}^{L, j}(B=0) & =J_{k^{\prime} k}^{L, j} \\
J_{k^{\prime} k}^{R, j}(B=0) & =J_{k^{\prime} k}^{R, j} \\
Q_{k^{\prime} k}^{j}(B=0) & =0
\end{aligned}
$$

The following symmetry relations have to be fulfilled during the flow due to the hermiticity of the Hamiltonian

$$
\begin{aligned}
J_{k^{\prime} k}^{\mathrm{sum}, j} & =J_{k k^{\prime}}^{\mathrm{sum}, j}, \\
P_{k^{\prime} k}^{j} & =M_{k k^{\prime}}^{j} .
\end{aligned}
$$

The interaction $P_{k^{\prime} k}^{j}$ refers to a scattering process involving a singlet to triplet transition as indicated in Eq. (17). The interaction $M_{k^{\prime} k}^{j}$ is the hermitian conjugate of $P_{k^{\prime} k}^{j}=\left(M_{k^{\prime} k}^{j}\right)^{\dagger}$. 


\section{Discussion of the leads}

In this paper we will concentrate on the case of two exchange coupled quantum dots which are not coupled symmetrically to a set of leads. We want to test the two quantum dots independently and therefore we assume that there are two leads attached to each quantum dot such that transport can take place through each quantum dot independently, see Fig. 1].

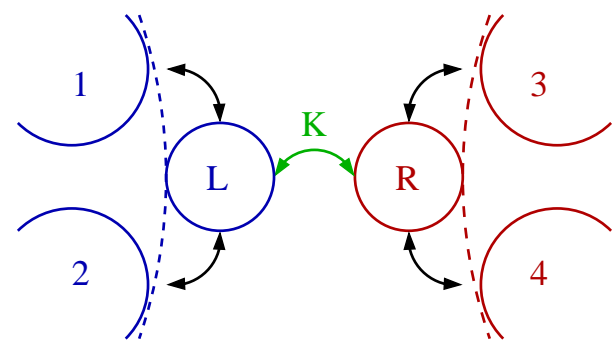

FIG. 1: Double Quantum Dot Setup: the residing electrons with a spin degree of freedom on the left (L) and right $(\mathrm{R})$ quantum dot are coupled mutually by the exchange interaction $K$. Two lead 1 and 2 (3 and 4) measure the current through the left (right) quantum dot. The leads are coupled to the quantum dots only by the Kondo spin exchange interaction. In the flow equation method we symmetrize the leads 1 and 2 (3 and 4 ) to one symmetric left (right) lead as denoted by dashed lines.

A completely symmetric setup corresponds to two Kondo impurities embedded in a metal, but in this case the two impurities couple to the same position in the lead and for example the RKKY interaction is not defined properly. On the other hand for completely symmetric coupling the initial conditions are $J_{k^{\prime} k}^{\text {sum, }}(B=0)=J_{0}$ and $P_{k^{\prime} k}^{j}(B=0)=M_{k^{\prime} k}^{j}(B=0)=0$. The singlet-triplet transitions are not allowed in the beginning and never created during the flow. Note that $\left[\left(S_{L}+S_{R}\right)^{2}, H\right]=0$ and thus the total spin is conserved. The flow yields the physics of a non-degenerate singlet or a spin-1 coupled to leads depending on the initial condition.

In order to make non-equilibrium electron transport possible, two leads have to be put at different chemical potential, $\mu_{1 / 2}= \pm e V_{L} / 2$ where 1 and 2 denote the two leads attached to the left dot; similarly 3 and 4 for the right two leads, $\mu_{3 / 4}= \pm e V_{R} / 2$. While we treat the four leads independently in the pRG approach, we can introduce a simplification due to symmetry arguments in the flow equation calculation.

We focus now for a short while on the left dot and leads 1 and 2 . It has been shown ${ }^{17}$, that a quantum dot is coupled only to the even mode of the two leads defined as

$$
c_{L}=\sqrt{\frac{r_{L}}{1+r_{L}}} c_{1}+\sqrt{\frac{1}{1+r_{L}}} c_{2}
$$

where $r_{L}=J_{11} / J_{22}\left(r_{R}=J_{33} / J_{44}\right)$ is the asymmetry parameter. For simplicity we only discuss $r_{L}=r_{R}=1$ in the numerics. Note that the extension to $r_{\alpha} \neq 1$ is trivial. Therefore we have to take into account only 2 instead of 4 leads but with a double step-like occupation function $n_{\alpha}(\epsilon)$

$$
n_{\alpha}(\epsilon)=\left\{\begin{array}{cl}
0 & \text { if } \epsilon>e V_{\alpha} / 2 \\
r_{\alpha} /\left(1+r_{\alpha}\right) & \text { if }|\epsilon|<e V_{\alpha} / 2 . \\
1 & \text { if } \epsilon<-e V_{\alpha} / 2
\end{array}\right.
$$

where $\alpha=L, R$ denotes the left or right set of leads. A non-zero voltage enters the calculation thus via the normal ordering of the lead electrons, see Ref. 6. In the following we assume that initially no cross-talk between the left dot and the right leads and vice versa is present, such that we can "drive" and "probe" the two quantum dots independently by each their leads. This corresponds to the initial conditions

$$
J_{k^{\prime} k}^{L R}(B=0)=J_{k^{\prime} k}^{R L}(B=0)=0 .
$$

Consequently the initial conditions read

$$
\begin{aligned}
J_{k^{\prime} k}^{L L}(B=0) & =J_{k^{\prime} k}^{R R}(B=0)=2 J_{0}, \\
\Rightarrow \quad J_{k^{\prime} k}^{\mathrm{sum}, L}(B=0) & =J_{k^{\prime} k}^{\mathrm{sum}, R}(B=0)=J_{0}, \\
P_{k^{\prime} k}^{L}(B=0) & =M_{k^{\prime} k}^{L}(B=0)=\frac{1}{2} J_{0}, \\
P_{k^{\prime} k}^{R}(B=0) & =M_{k^{\prime} k}^{R}(B=0)=-\frac{1}{2} J_{0},
\end{aligned}
$$

where we assumed the symmetry that the left and right coupling are equal to the fixed but arbitrary value $J_{0}$. Note that this model has been studied in detail by one of the authors in Ref. 14 where the system showed a current through the left dot even when voltage was applied on the right side (transconductance effect) as discussed in more detail at the end of this work.

\section{FLOW EQUATIONS IN LOWEST ORDER}

\section{A. The method of flow equations}

In a model with a clear separation of energy scales it is often an advantage to focus on the low-energy physics and find an effective representation for the high-energy physics. In the poor man's scaling approach as will also be discussed in more detail in the next section, the scattering processes containing energies at the large band edges are integrated out. Their effective contribution is put into a renormalized interaction and by further reducing the band cutoff one arrives at an effective model for a low-energy band.

The separation of energy scales is also important in the flow equation method. The philosophy here is again to find an effective Hamiltonian which describes the same physics as the original model but can be easily solved like a quadratic Hamiltonian. As an example the kinetic Hamiltonian $\epsilon_{k \sigma} c_{k \sigma}^{\dagger} c_{k \sigma}$ is diagonal in the conduction electron momenta, whereas the interaction part proportional 
to $: \vec{s}_{k^{\prime} k}=\frac{1}{2} c_{k^{\prime} \sigma^{\prime}}^{\dagger} \vec{\tau}_{\sigma^{\prime} \sigma} c_{k \sigma}$ connects electrons with different momenta. In the matrix representation of the Hamiltonian $H$ we separate the diagonal contributions, $H_{0}$, from the off-diagonal contributions $H_{\text {int }}$. The aim of the flow equation method is to generate an effective $H(B)$ starting with some general flow parameter $B=0$ and modify $H(B)$ accordingly such that $H(B=\infty)$ is diagonal.

In the flow equation method we achieve this by infinitesimal unitary transformations ${ }^{21}$

$$
\frac{d}{d B} H(B)=[\eta(B), H(B)] .
$$

Note that this expression forces the generator $\eta=-\eta^{\dagger}$ to be anti-hermitian which is equivalent to claiming that the transformation is unitary. The choice of the generator can be different from problem to problem, but the canonical generator 21

$$
\eta(B)=\left[H_{0}, H_{\text {int }}(B)\right]
$$

has proven to be a stable choice. The canonical generator automatically fulfills $\eta^{\dagger}=-\eta$. Since it is the product of two Hamiltonians it is proportional to energy ${ }^{2}$. Consequently the flow parameter $B$ is of the order of $1 /$ energy $^{2}$.

In contrast to the standard scaling theories this choice of rescaling eliminates the outermost components in the matrix Hamiltonian or in other words: scattering processes involving an energy transfer of the order of the frequency band-cutoff $\Lambda$ are integrated out in the course of the flow procedure, $B=1 / \Lambda^{2}$. The final Hamiltonian contains only energy-diagonal processes with a renormalized energy. It is a clear advantage to keep information on all energy scales, in particular for the non-equilibrium situation where scattering processes away from the ground state play an important role.

For a more extended introduction to the flow equation method we refer to Ref. 17. As a side remark we want to mention that corrections from taking into account normal ordering with respect to the interacting ground state are of fourth order in the interaction ${ }^{7}$ and can thus safely be neglected in our calculation to third order. A first application of the flow equation method to coupled quantum dots in equilibrium can be found in Ref. 22 .

\section{B. Flow equation for the double quantum dot system}

For the double quantum dot system studied in this paper the generator $\eta$ is chosen to be the canonical generator $\eta=\left[H_{0}, H_{\text {int }}\right]$ and given explicitly by

$$
\begin{aligned}
& \eta=\sum_{j=L, R} \sum_{k^{\prime} k} \eta_{k^{\prime} k}^{\mathrm{sum}, j}:\left(\vec{S}_{L}+\vec{S}_{R}\right) \vec{s}_{\left(k^{\prime} j\right)(k j)} \\
& +\sum_{j=L, R} \sum_{k^{\prime} k} \eta_{k^{\prime} k}^{P / M, j}:\left(\left(\vec{S}_{L}-\vec{S}_{R}\right) \pm 2 i\left(\vec{S}_{L} \times \vec{S}_{R}\right)\right) \vec{s}_{\left(k^{\prime} j\right)(k j)}:
\end{aligned}
$$

where

$$
\begin{aligned}
\eta_{k^{\prime} k}^{\mathrm{sum}, j} & =\left(\epsilon_{k^{\prime}}-\epsilon_{k}\right) J_{k^{\prime} k}^{\mathrm{sum}, j}, \\
\eta_{k^{\prime} k}^{P j} & =\left(\epsilon_{k^{\prime}}-\epsilon_{k}+K\right) P_{k^{\prime} k}^{j}, \\
\eta_{k^{\prime} k}^{M j} & =\left(\epsilon_{k^{\prime}}-\epsilon_{k}-K\right) M_{k^{\prime} k}^{j}
\end{aligned}
$$

As discussed before we can here observe that the coupling $P_{k^{\prime} k}^{j}$ or $M_{k^{\prime} k}^{j}$ corresponds to a transition between a singlet and triplet state with an energy cost of $\pm K$, respectively.

Due to the construction of the canonical generator $(\propto$ energy $^{2}$ ) the flow parameter $B$ is related to the traditional energy/frequency cutoff $\Lambda$ by

$$
B \propto \frac{1}{\Lambda^{2}} .
$$

Inserting the canonical generator $\eta$ into the flow equation, Eq. (35), we find in lowest, linear order an exponential behavior of the coupling functions. Thus we can define an effective coupling $\overline{J_{k^{\prime} k}^{\text {sum }, j}}(B)$

$$
J_{k^{\prime} k}^{\mathrm{sum}, j}(B)=\mathrm{e}^{-B\left(\epsilon_{k^{\prime}}-\epsilon_{k}\right)^{2}} \overline{J_{k^{\prime} k}^{\mathrm{sum}, j}}(B) .
$$

and

$$
\begin{aligned}
P_{k^{\prime} k}^{j}(B) & =\mathrm{e}^{-B\left(\epsilon_{k^{\prime}}-\epsilon_{k}+K\right)^{2}} \overline{P_{k^{\prime} k}^{j}}(B), \\
M_{k^{\prime} k}^{j}(B)=P_{k k^{\prime}}^{j}(B) & =\mathrm{e}^{-B\left(\epsilon_{k^{\prime}}-\epsilon_{k}-K\right)^{2}} \overline{P_{k k^{\prime}}^{j}}(B),
\end{aligned}
$$

The effective couplings $\overline{J_{k^{\prime} k}^{\text {sum, } j}}$ and $\bar{P}_{k^{\prime} k}^{j}$ obey a scaling equation with a scaling function $\beta$ which has to be determined from higher than linear order terms. The exponential dependence though mirrors the physical picture of the Kondo coupling: It is logarithmically divergent when energy scattering processes with initial state $k$ and final state $k^{\prime}$ are energy-degenerate, e.g. $\mathrm{e}^{-\left(\left(\epsilon_{k^{\prime}}-\epsilon_{k}\right) / \Lambda\right)^{2}}=1$ for $\epsilon_{k^{\prime}}=\epsilon_{k}$, and away from the coherence conditions the coupling functions are suppressed, see also Fig. 2 and corresponding discussion. In contrast to the single-impurity Kondo model (without magnetic field), a divergent coupling for $P_{k^{\prime} k}^{j}$ representing the singlet-triplet transition can only be expected when a scattering process in the leads matches the energy of a transition inside the quantum dot.

The full expression for the flow equation calculation to second order in the interaction, the so-called one-loop order, is given in the appendix. In Fig. 2 we show full numerical calculations for these one-loop result in the case of $k^{\prime}=k$ and compare with the solution obtained by the diagonal parametrization 7.8 . This is a by now wellestablished approximation that allows some analytic insight into the flow equations and simplifies the numerical effort significantly.

In the diagonal parametrization we assume that the important energy dependence $\epsilon_{k}$ (momentum $k$ ) is given by the exponential decay $e^{-B\left(\epsilon_{k^{\prime}}-\epsilon_{k}+\alpha K\right)^{2}}$ and we can approximate

$$
e^{-B\left(\epsilon_{k^{\prime}}-\epsilon_{k}+\alpha K\right)^{2}} f\left(\epsilon_{k^{\prime}}, \epsilon_{k}\right) \approx e^{-B\left(\epsilon_{k^{\prime}}-\epsilon_{k}+\alpha K\right)^{2}} f\left(\epsilon_{\Sigma}\right),
$$


where $\alpha=\{0, \pm 1\}$ and $\epsilon_{\Sigma}=\left(\epsilon_{k^{\prime}}+\epsilon_{k}\right) / 2$.

Starting from two energy arguments for the incoming and outgoing conduction electron one energy is kept fixed but arbitrary and the other is assumed to fulfill the equation $\epsilon_{k^{\prime}}-\epsilon_{k}+\alpha K=0$. For example for the coupling to the total spin $\left(\vec{S}_{L}+\vec{S}_{R}\right)$ this yields:

$$
\begin{aligned}
& J_{k^{\prime} k}^{\mathrm{sum}, j}(B)=\mathrm{e}^{-B\left(\epsilon_{k^{\prime}}-\epsilon_{k}\right)^{2}} J_{\left(k^{\prime}+k\right) / 2}^{\mathrm{sum}, j}(B) \\
& \text { where } J_{k}^{\mathrm{sum}, j}(B):=J_{k, k}^{\mathrm{sum}, j}(B) .
\end{aligned}
$$

In the diagonal parametrization for $P_{k^{\prime} k}^{j} / M_{k^{\prime} k}^{j}$ one has to be cautious since the choice $\epsilon_{k^{\prime}}-\epsilon_{k}=-K$ in $P_{k^{\prime} k}^{j}$ is not unique. The correct momentum dependence is only recovered if we choose the diagonal parametrization as

$$
\begin{aligned}
P_{k^{\prime} k}^{j}(B) & =\mathrm{e}^{-B\left(\epsilon_{k^{\prime}}-\epsilon_{k}+K\right)^{2}} P_{\left(k^{\prime}+k\right) / 2}^{j}(B) . \\
\text { where } \quad P_{k}^{j}(B) & :=P_{\epsilon_{k}-K / 2, \epsilon_{k}+K / 2}^{j}(B)
\end{aligned}
$$

Note that in the definition of $P_{k}^{j}$ the average energy $\epsilon_{\Sigma}$ is given by $\epsilon_{\Sigma}=\left(\epsilon_{k}-K / 2+\epsilon_{k}+K / 2\right) / 2=\epsilon_{k}$. For the coupling $M_{k^{\prime} k}^{j}=P_{k k^{\prime}}^{j}$ the assumption in Eq. (45), i.e. $\epsilon_{k^{\prime}}-K / 2=\epsilon_{k}+K / 2$, is automatically fulfilled and in diagonal parametrization:

$$
\begin{aligned}
M_{k^{\prime} k}^{j}(B) & =\mathrm{e}^{-B\left(\epsilon_{k^{\prime}}-\epsilon_{k}-K\right)^{2}} P_{\left(k^{\prime}+k\right) / 2}^{j}(B) \\
\text { since } & \\
P_{k}^{j}(B) & =M_{\epsilon_{k}+K / 2, \epsilon_{k}-K / 2}^{j}(B)=P_{\epsilon_{k}-K / 2, \epsilon_{k}+K / 2}^{j}(B) .
\end{aligned}
$$

Using the assumption in Eq. (45) which leads to the diagonal parametrization we arrive at the one-loop flow equations

$$
\begin{aligned}
& \frac{d J_{k}^{\mathrm{sum}, j}(B)}{d B}= \\
& -\sum_{q}(1-2 n(q j))\left(\epsilon_{k}-\epsilon_{q}\right) \mathrm{e}^{-2 B\left(\epsilon_{k}-\epsilon_{q}\right)^{2}}\left(J_{(k+q) / 2}^{\mathrm{sum}, j}\right)^{2} \\
& -4 \sum_{q}(1-n(q j))\left(\epsilon_{k}-\epsilon_{q}+K\right) \mathrm{e}^{-2 B\left(\epsilon_{k}-\epsilon_{q}+K\right)^{2}}\left(P_{(k+q) / 2}^{j}\right)^{2} \\
& +4 \sum_{q} n(q j)\left(\epsilon_{k}-\epsilon_{q}-K\right) \mathrm{e}^{-2 B\left(\epsilon_{k}-\epsilon_{q}-K\right)^{2}}\left(P_{(k+q) / 2}^{j}\right)^{2}
\end{aligned}
$$

and

$$
\begin{aligned}
& \frac{d P_{k}^{j}(B)}{d B}= \\
& -\sum_{q}(1-n(q j))\left(2\left(\epsilon_{k}-\epsilon_{q}-K / 2\right)\right) \mathrm{e}^{-2 B\left(\epsilon_{k}-\epsilon_{q}-K / 2\right)^{2}} \\
& \quad J_{(k-K / 2+q) / 2}^{\text {sum }, j} P_{(q+k+K / 2) / 2}^{j} \\
& +\sum_{q} n(q j)\left(2\left(\epsilon_{k}-\epsilon_{q}+K / 2\right)\right) \mathrm{e}^{-B\left(\epsilon_{k}-\epsilon_{q}+K / 2\right)^{2}} \\
& P_{(k-K / 2+q) / 2}^{j} J_{(q+k+K / 2) / 2}^{\mathrm{sum}, j}
\end{aligned}
$$

For details of the calculation we refer to the appendix $\mathrm{A}$. Note that $M_{k^{\prime} k}^{j}$ is given immediately by the solution for $P_{k}^{j}$ in the diagonal parametrization.

The one-loop order contains the integration over one internal degree of freedom, the momentum $q$. Assuming a constant density of states (DOS), $N(0)=1 /\left(2 \Lambda_{0}\right)$, of a flat band with bandwidth $\Lambda_{0}$ around the Fermi energy, we transform the summation over momenta $q$ to an integral over the energy $\epsilon_{q}: \sum_{q} \rightarrow N(0) \int_{-\Lambda_{0}}^{\Lambda_{0}} d \epsilon_{q}$. The DOS is absorbed into the dimensionless couplings $g_{k^{\prime} k}^{\text {sum }, j}=N(0) J_{k^{\prime} k}^{\text {sum }, j}$ and $p_{k^{\prime} k}^{j}=N(0) P_{k^{\prime} k}^{j}$.

Predicting that the couplings will be only logarithmically dependent on the energy we assume that the energy dependence is dominated by the exponential function and simplify

$$
f(x) \exp \left(-2 B(x-c)^{2}\right) \approx f(c) \exp \left(-2 B(x-c)^{2}\right)
$$

Then the integration in Eqs. (52) and (53) is independent of the energy argument of the coupling function. Straightforwardly we find for example

$$
\begin{gathered}
\int d \epsilon_{q}(1-2 n(q j))\left(\epsilon_{k}-\epsilon_{q}+\alpha K\right) \mathrm{e}^{-2 B\left(\epsilon_{k}-\epsilon_{q}+\alpha K\right)^{2}} \\
=-\frac{1}{2 B} N(0)\left[\frac{r_{j}}{1+r_{j}} e^{-2 B\left(\epsilon_{k}-V_{j} / 2+\alpha K\right)^{2}}\right. \\
\left.+\frac{1}{1+r_{j}} e^{-2 B\left(\epsilon_{k}+V_{j} / 2+\alpha K\right)^{2}}\right]
\end{gathered}
$$

where we used the two-step Fermi function, Eq. (29), and $\alpha$ as placeholder for the corresponding prefactor to $K$. If we assume that the leads are symmetrically coupled (asymmetry parameter $r_{j}=1$ ) we can write the flow equations as

$$
\begin{aligned}
\frac{d g_{k}^{\text {sum }, j}(B)}{d B}= & \frac{1}{2 B} \sum_{\nu= \pm 1} \frac{1}{2} \mathrm{e}^{-2 B\left(\epsilon_{k}+\nu V_{j} / 2\right)^{2}}\left(g_{k}^{\text {sum }, j}\right)^{2} \\
& +2 \frac{1}{2 B} \sum_{\nu= \pm 1} \frac{1}{2} \mathrm{e}^{-2 B\left(\epsilon_{k}+K+\nu V_{j} / 2\right)^{2}}\left(p_{k+K / 2}^{j}\right)^{2} \\
& +2 \frac{1}{2 B} \sum_{\nu= \pm 1} \frac{1}{2} \mathrm{e}^{-2 B\left(\epsilon_{k}-K+\nu V_{j} / 2\right)^{2}}\left(p_{k-K / 2}^{j}\right)^{2}
\end{aligned}
$$

and

$$
\begin{aligned}
\frac{d p_{k}^{j}(B)}{d B}= & \frac{1}{2 B} \sum_{\nu= \pm 1} \frac{1}{2} \mathrm{e}^{-2 B\left(\epsilon_{k}-K / 2+\nu V_{j} / 2\right)^{2}} g_{k-K / 2}^{\text {sum }, j} p_{k}^{j} \\
& +\frac{1}{2 B} \sum_{\nu= \pm 1} \frac{1}{2} \mathrm{e}^{-2 B\left(\epsilon_{k}+K / 2+\nu V_{j} / 2\right)^{2}} p_{k}^{j} g_{k+K / 2}^{\text {sum }, j}
\end{aligned}
$$

Note that here the term at $\epsilon_{k}=0$ is exponentially small for $B \gg\left(V_{j} / 2\right)^{2}$ due to $\mathrm{e}^{-B\left(V_{j} / 2\right)^{2}}$ (assuming $K=0$ ). Reducing the band cutoff $\Lambda$ to 0 in a system with a large applied voltage thus leads to a failure of the theory. If the voltage is taken beyond the linear response regime 


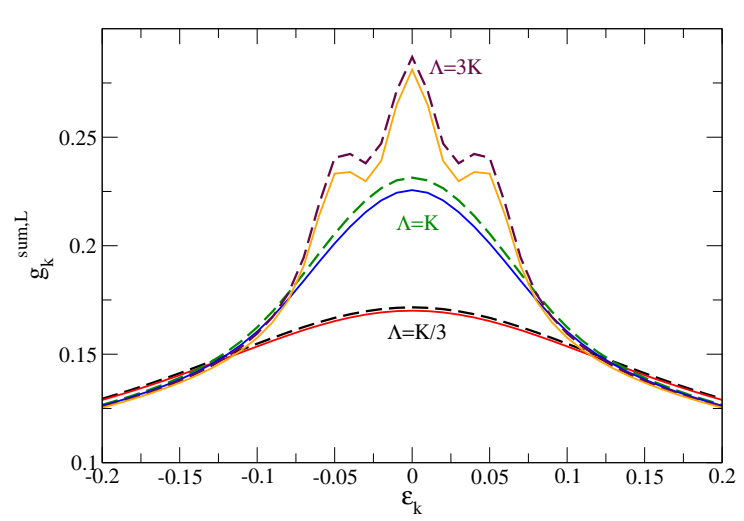

FIG. 2: Flow of the coupling $g_{k^{\prime} k}^{\text {sum, } L}$ for $\epsilon_{k^{\prime}}=\epsilon_{k}$ in the numerical exact calculation (dashed lines) and for $g_{k}^{\text {sum }}$ in the diagonal parametrization (solid lines) versus energy $\epsilon_{k}$ for three different values $B=1 / \Lambda^{2}$ and $\Lambda=K / 3, K, 3 K$. Further parameters of this plot are chosen $K / 2 \Lambda_{0}=0.05$, and $g\left(\Lambda_{0}\right)=0.128$ leading to $T_{K}=0.01$. Note that the flow of $K$ is neglected here.

it is necessary to study the frequency/energy-dependent behavior of the coupling functions and the divergence at the two new Fermi edges $\epsilon_{k}= \pm V_{j} / 2$.

\section{Discussion of results}

Note that the left and right leads do not mix in Eqs. (56) and (57) and therefore the left and right coupling can be studied individually.

In Fig. 2 the flow of the coupling $g_{k^{\prime} k}^{\text {sum, } L}$ is plotted for $\epsilon_{k}=\epsilon_{k^{\prime}}$ versus the energy $\epsilon_{k}$ for different values of the flow parameter $B$ while the voltage $V$ is set to zero. Since there is no cross-coupling the scaling behavior of $g_{k^{\prime} k}^{\text {sum }, R}$ is identical to $g_{k^{\prime} k}^{\text {sum, } L}$. The initial value of $g_{k^{\prime} k}^{\text {sum, } L}$ is energy-independent but very soon a frequency dependence is produced, and for large enough $B$ peaks at the Fermi edge $\epsilon_{k}=0$ and at non-zero energy $\epsilon_{k}= \pm K$ are visible. Values away from $\epsilon_{k}=0, \pm K$ are exponentially suppressed. As obvious from Fig. 2 the diagonal parametrization $g_{k}^{\text {sum, } L}$ reproduces the frequency dependence of $g_{k^{\prime} k}^{\text {sum, } L}$ perfectly and also the absolute value of the coupling is in very good agreement. Thus we can conclude from the numerical comparison in Fig. 2 that the diagonal parametrization is a good approximation.

This has proven to be the case for most problems studied previously in the context of non-equilibrium Kondo models solved with the flow equation method 7.8 . In the following we will also show that the diagonal parametrization is equivalent to the non-equilibrium scaling method by A. Rosch et al. $\stackrel{2}{2}$ and can thus also confirm the accuracy and the validity of the assumptions in the other approach.

For $B \ll \min \left[1 / K^{2}, 1 / \epsilon_{k}^{2}, 1 / V_{j}^{2}\right]$ the scaling equation for $g_{k}^{\text {sum }, j}$ and $p_{k}^{j}$ are identical since the exponential $\mathrm{e}^{-2 B\left(\epsilon_{k}+\alpha K+\nu V_{j} / 2\right)^{2}} \approx 1$ does not cutoff the flow. Note though, that the initial value of $p_{k}^{j}\left(B_{0}\right)=1 / 2 g_{k}^{\text {sum }, j}\left(B_{0}\right)$. Using $B=1 / \Lambda^{2}$ where $\Lambda$ is the frequency cutoff the flow equation (56) thus simplifies to the well-known Kondo coupling scaling function for a spin- $1 / 2$,

$$
\frac{d g}{d \ln \Lambda}=-2 g^{2} .
$$

This equation gives us a one-loop Kondo temperature of

$$
T_{K}=\Lambda_{0} \mathrm{e}^{-1 / 2 g\left(\Lambda_{0}\right)} .
$$

Thus we expect the flow equation (58) to diverge and the lowest order to break down when $B$ reaches the value $1 / T_{K}^{2}$. Nonzero temperature and current induced decoherence can remove this divergence as we will explain later on. However, first we want to introduce the perturbative RG approach in the next section.

\section{GENERALIZED PERTURBATIVE RG}

The interaction of a Kondo problem has the general structure

$$
H_{\mathrm{int}}=\sum_{\substack{n \sigma, m \sigma^{\prime} \\ \gamma, \gamma^{\prime}}} \frac{1}{4}\left(\vec{T}_{\alpha}\right)_{\gamma^{\prime} \gamma} \vec{\tau}_{\sigma^{\prime} \sigma} J_{\gamma, \omega_{\gamma} ; \gamma^{\prime}, \omega_{\gamma^{\prime}}}^{n \sigma, \omega_{c} ; m \omega^{\prime}, \omega_{c}^{\prime}} d_{\gamma^{\prime}}^{\dagger} d_{\gamma} c_{m \sigma^{\prime}}^{\dagger} c_{n \sigma},
$$

where $\gamma, \gamma^{\prime}$ refer to the eigenstates of the eigenstates of the double quantum dot (singlet and triplet in this example), $m, n$ are the lead indices, $\sigma, \sigma^{\prime}$ are spin up and down states and $\vec{\tau}_{\sigma^{\prime} \sigma}$ refers to the Pauli spin matrix and $\vec{T}_{\gamma^{\prime} \gamma}$ is a generalized Pauli matrix which has to be chosen in accordance with the internal structure of the quantum dot setup. The momentum dependence of the interaction is neglected and thus we write the Kondo interaction in terms of the momentum integrated conduction electron operators, $c_{n \sigma}=\sum_{k} c_{n k \sigma}$.

This Hamiltonian can be furthermore described by a general vertex

$$
\mathcal{V}_{\gamma, \omega_{\gamma} ; \gamma^{\prime}, \omega_{\gamma^{\prime}}}^{n \sigma, \omega_{c} ; m \sigma^{\prime}, \omega_{c}^{\prime}}=\frac{1}{4} \vec{\tau}_{\sigma^{\prime} \sigma}\left(\vec{T}_{\alpha}\right)_{\gamma^{\prime} \gamma} N(0) J_{\gamma, \omega_{\gamma} ; \gamma^{\prime}, \omega_{\gamma^{\prime}}}^{n \sigma, \omega_{c} ; m \sigma^{\prime}, \omega_{c}^{\prime}} .
$$

Note that the Hamiltonian necessarily has to be hermitian and thus

$$
\mathcal{V}_{\gamma, \omega_{\gamma} ; \gamma^{\prime}, \omega_{\gamma^{\prime}}}^{n \sigma, \omega_{c} ; m \sigma^{\prime}, \omega_{c}^{\prime}}=\mathcal{V}_{\gamma^{\prime}, \omega_{\gamma^{\prime}} ; \gamma, \omega_{\gamma}}^{m \sigma^{\prime}, \omega_{c}^{\prime} ; n \sigma \omega_{c}}
$$

if all couplings are real numbers.

\section{A. General scaling equation}

The idea of scaling has already been introduced. Instead of calculating a perturbative series of a physical 
quantity, we do a perturbation in the couplings of the interaction. This provides us with results beyond standard perturbation theory, which is known to fail in the Kondo model.

One of the first scaling theories was introduced as "poor man's" scaling by P. W. Anderson in Ref. 23 and generalized to non-equilibrium by A. Rosch et al. ${ }^{2}$. Importantly, the physics of the problem at hand, i.e. the expectation value of any physical observable, needs to be invariant under scaling. Anderson studied the T-matrix for the Kondo model and showed that a scattering into a high-energy state can be absorbed into a lower-energy setup by including the process to the bandedge $\Lambda$ and back into an effective interaction for a smaller bandwidth $\Lambda-d \Lambda$. There are two contributions equivalent to a scattering process to the upper band edge (electron $=$ Cooper contribution) and lower band edge (hole = Peierls contribution $)^{3}$. By successively reducing the band cutoff $\Lambda$ in infinitesimal steps $d \Lambda$ we generate an effective interaction at low energy scales which is of the same form as the original one but contains a renormalized Kondo coupling $J(\Lambda)$. The change is described by a scaling equation $d J(\Lambda) / d \Lambda$ and including only one virtual state at the band edge is referred to as one-loop order. As the poor man's scaling is also perturbative, a truncation has to be made and only certain renormalization diagrams are taken into account.

In the traditional poor man's scaling ${ }^{23}$ the band cutoff is reduced to zero, $\Lambda \rightarrow 0$. This is a problem in a non-equilibrium situation as emphasized before because energies beyond the ground state play an important role. In Ref. 2 the approach from Anderson was generalized to renormalize only one of the two band-cutoffs, e.g. the outgoing energy $\hbar \omega_{c}^{\prime}$ for a vertex while the incoming en- ergy $\hbar w_{c}$ is fixed (but arbitrary). In this context the Cooper and Peierls contribution to the scaling equation have to be calculated in Keldysh notation and we find ${ }^{15}$ that the leading logarithmic contribution originates from an integral of the form

$$
\frac{\partial}{\partial \ln \Lambda} \int_{-\Lambda}^{\Lambda} d \epsilon \frac{1}{x-\epsilon} \tanh \left(\frac{\epsilon}{2 T}\right) \approx-\frac{\Lambda}{x-\Lambda}
$$

i.e. the real part of the quantum dot Green's function and the lesser part of the conduction electron Green's function including the sharp step of the Fermi function at zero temperature. The external energy $x$ can be the exchange energy $K$, magnetic field $h$, chemical potential $\mu$ or combinations of those.

In the perturbative $\mathrm{RG}$ method we approximate $\Lambda /(\Lambda-x)$ by $\Theta(\Lambda-|x|)$ since $\Lambda /(\Lambda-x) \approx 1$ for $\Lambda \gg x$ and $\Lambda /(\Lambda-x) \approx-\Lambda / x \approx 0$ for $x \gg \Lambda$. At this point we like to state that one major difference between the two scaling method is the choice of the cutoff-function. In the flow equation method the cutoff function $\mathrm{e}^{-B x^{2}}$ is valid in general and therefore we expect a better resolution in the proximity of the logarithmically enhanced peaks. For further information on the $\mathrm{pRG}$ method we refer the interested reader to Ref. 3 or 15 .

In the general notation

$$
H_{\mathrm{int}}=\sum_{\gamma \gamma^{\prime}} \sum_{n \sigma ; m \sigma^{\prime}} \mathcal{V}_{\gamma, \omega_{\gamma} ; \gamma^{\prime}, \omega_{\gamma^{\prime}}}^{n \sigma, \omega_{c} ; m \sigma^{\prime}, \omega_{c}^{\prime}} d_{\gamma^{\prime}}^{\dagger} d_{\gamma} c_{m \sigma^{\prime}}^{\dagger} c_{n \sigma}
$$

one can derive a scaling equation of the form

$$
\begin{aligned}
\frac{\partial \mathcal{V}_{\gamma, \omega_{\gamma} ; \gamma^{\prime}, \omega_{\gamma^{\prime}}}^{n \sigma, \omega_{c} ; m \sigma^{\prime}, \omega_{c}^{\prime}}}{\partial \ln \Lambda}=\frac{1}{2} \sum_{\lambda= \pm 1} \sum_{\eta, \nu, s} & \left(\mathcal{V}_{\eta, \omega_{\eta} ; \gamma^{\prime}, \omega_{\gamma^{\prime}}}^{\nu s ; \lambda \Lambda+\mu_{\nu} ; m \sigma^{\prime}, \omega_{c}^{\prime}} \Theta_{\left|\omega_{c}+\omega_{\gamma}-\mu_{\nu}-\epsilon_{\eta}\right|} \mathcal{V}_{\gamma, \omega_{\gamma} ; \eta, \omega_{\eta}}^{n \sigma, \omega_{i} ; \nu s, \lambda \Lambda+\mu_{\nu}}\right. \\
& \left.-\mathcal{V}_{\eta, \omega_{\eta} ; \gamma^{\prime}, \omega_{\gamma^{\prime}}}^{n \sigma, \omega_{c} ; \nu s, \lambda \Lambda+\mu_{\nu}} \Theta_{\left|\omega_{\gamma^{\prime}}-\omega_{c}+\mu_{\nu}-\epsilon_{\eta}\right|} \mathcal{V}_{\gamma, \omega_{\gamma} ; \eta, \omega_{\eta}}^{\nu s, \lambda \Lambda+\mu^{\prime}, \omega_{c}^{\prime}}\right)
\end{aligned}
$$

where we introduced the notation $\Theta_{x}=\Theta(\Lambda-|x|)$. Eq. (65) is the generalization of the equations given in Ref. 2. A derivation can be found in Ref. 15. Every ingoing and outcoming leg of the vertex is assigned with a frequency, $\omega_{c}, \omega_{c}^{\prime}$ and $\omega_{\gamma}, \omega_{\gamma^{\prime}}$ for the conduction electron and quantum dot pseudo fermions, respectively.

\section{B. pRG for the Double Quantum Dot System}

We now concentrate on the case of the double quantum dot system where the general vertex is of the form
Eq. (61). Furthermore we assume that there is no external magnetic field applied and therefore the setup is spatially invariant, i.e. the three triplet states $t_{+}, t_{0}, t_{-}$ are degenerate. Like in the flow equation approach there are thus only three vertices: a triplet-triplet transition without energy cost/gain in the DQD, a singlet-triplet and a triplet-singlet transition involving such a process.

The general vertex has four frequencies assigned to it, where one frequency is fixed due to energy conservation, which we impose on the vertex. The quantum dot is described by pseudo-particles $d_{\gamma}$ which have to obey a constraint. In the following we set the energies "on-shell", 
e.g. the frequency of the incoming particle $\omega_{\gamma}$ is given by the eigenenergy of the state $\gamma$, i.e. $\epsilon_{\gamma}$ neglecting a finite lifetime due to hybridization with the leads:

$$
\mathcal{V}_{\gamma, \omega_{\gamma} ; \gamma^{\prime}, \omega_{\gamma^{\prime}}}^{n \sigma, \omega_{c} ; m \sigma^{\prime}, \omega_{c}^{\prime}} \approx \mathcal{V}_{\gamma, \epsilon_{\gamma} ; \gamma^{\prime}, \epsilon_{\gamma^{\prime}}}^{n \sigma, \omega_{c} ; m \sigma^{\prime}, \omega_{c}^{\prime}}
$$

With the energy conservation the vertex thus only depends on one frequency which is chosen to be the ingoing frequency in the following

$$
\mathcal{V}_{\gamma, \epsilon_{\gamma} ; \gamma^{\prime}, \epsilon_{\gamma^{\prime}}}^{n \sigma, \omega_{c} ; \omega_{c}^{\prime}}=\mathcal{V}_{\gamma \gamma^{\prime}}^{n \sigma ; m \sigma^{\prime}}\left(\omega_{c}\right)
$$

Evaluating now the product of Pauli matrices we arrive at the three scaling equations for the dimensionless Kondo couplings $g_{\gamma \gamma^{\prime}}^{n m}(\omega)=N(0) J_{\gamma \gamma^{\prime}}^{n m}(\omega)$ for zero magnetic field

$$
\begin{gathered}
\frac{\partial g_{t s}^{n m}(\omega)}{\partial \ln \Lambda}=-\frac{1}{2} \sum_{\nu}\left(2 g_{t s}^{\nu m}(\omega) g_{t t}^{n \nu}(\omega) \Theta_{\omega-\mu_{\nu}}\right. \\
\left.+2 g_{t s}^{n \nu}(\omega) g_{t t}^{\nu m}(\omega+K) \Theta_{\omega-\mu_{\nu}+K}\right), \\
\frac{\partial g_{s t}^{n m}(\omega)}{\partial \ln \Lambda}=-\frac{1}{2} \sum_{\nu}\left(2 g_{t t}^{\nu m}(\omega-K) g_{s t}^{n \nu}(\omega) \Theta_{\omega-\mu_{\nu}-K}\right. \\
\left.\quad+2 g_{t t}^{n \nu}(\omega) g_{s t}^{\nu m}(\omega) \Theta_{\omega-\mu_{\nu}}\right) \\
\frac{\partial g_{t t}^{n m}(\omega)}{\partial \ln \Lambda}=-\frac{1}{2} \sum_{\nu}\left(g_{s t}^{\nu m}(\omega+K) g_{t s}^{n \nu}(\omega) \Theta_{\omega-\mu_{\nu}+K}\right. \\
+g_{s t}^{n \nu}(\omega) g_{t s}^{\nu m}(\omega-K) \Theta_{\omega-\mu_{\nu}-K} \\
\left.+2 g_{t t}^{\nu m}(\omega) g_{t t}^{n \nu}(\omega) \Theta_{\omega-\mu_{\nu}}\right) .
\end{gathered}
$$

See reference 15 for the details of the derivation.

Note that in order to arrive at this scaling equations the following approximations had to be made. First, the cutoff is sent to 0 on the right hand side of Eq. (65). Otherwise the imposed energy conservation assumed for the left hand side is not fulfilled on the right hand side and the RG equation is not self-consistent. Second, the pseudo-fermions describing the quantum dots states are assumed to be on-shell, Eq. (66). This step is not necessary in the flow equation approach since the impurity spin is kept as an operator without introducing pseudo particles. Third, the energy conservation on the vertex is enforced and thus we end up with only one energy index (which is chosen to be the ingoing energy). Fourth, the approximations in Eqs. (45) and (54) are used in the integration identical to the approximations used in the flow equation method.

Note that the hermiticity of the Hamiltonian which leads to $p_{k^{\prime} k}=m_{k k^{\prime}}$ corresponds to

$$
g_{s t}^{m n}(\omega)=g_{t s}^{n m}(\omega-K)
$$

in the perturbative scaling approach.

In order to be able to compare the two results we introduce a new symmetrized coupling $\tilde{g}_{s t}^{n m}(\omega)$ analogous to the diagonal parametrization of $p_{k}^{j}$ which is defined as

$$
\begin{aligned}
& g_{s t}^{n m}(\omega)=2 \tilde{g}_{s t}^{n m}(\omega-K / 2), \\
& g_{t s}^{n m}(\omega)=2 \tilde{g}_{s t}^{n m}(\omega+K / 2),
\end{aligned}
$$

which thus fulfills the same initial condition as $p_{k}^{j}$

$$
\left.\tilde{g}_{s t}^{n m}(\omega)\right|_{\Lambda=\Lambda_{0}}=\left.\frac{1}{2} g_{s t}^{n m}(\omega+K / 2)\right|_{\Lambda=\Lambda_{0}}=\frac{1}{2} N(0) J_{0}
$$

and is peaked at $\pm K / 2$ instead of at $0, K$ and $-K, 0$ where $g_{s t}^{n m}$ and $g_{t s}^{n m}$ show resonant features.

With this new definition and inserting the approximations as mentioned above yields the scaling equations

$$
\begin{aligned}
\frac{\partial \tilde{g}_{s t}^{n m}(\omega)}{\partial \ln \Lambda} & =-\sum_{\nu}\left(g_{t t}^{\nu m}(\omega+K / 2) \tilde{g}_{s t}^{n \nu}(\omega) \Theta_{\omega-\mu_{\nu}-K / 2}\right. \\
& \left.+g_{t t}^{n \nu}(\omega-K / 2) \tilde{g}_{s t}^{\nu m}(\omega) \Theta_{\omega-\mu_{\nu}+K / 2}\right) \\
\frac{\partial g_{t t}^{n m}(\omega)}{\partial \ln \Lambda} & =-\sum_{\nu}\left(2 \tilde{g}_{s t}^{\nu m}(\omega+K / 2) \tilde{g}_{s t}^{n \nu}(\omega+K / 2) \Theta_{\omega-\mu_{\nu}+K}\right. \\
& +2 \tilde{g}_{s t}^{n \nu}(\omega-K / 2) \tilde{g}_{s t}^{\nu m}(\omega-K / 2) \Theta_{\omega-\mu_{\nu}-K} \\
& \left.+g_{t t}^{\nu m}(\omega) g_{t t}^{n \nu}(\omega) \Theta_{\omega-\mu_{\nu}}\right)
\end{aligned}
$$

In most applications a further convenient approximation is used: in the frequency integral over all coupling functions it is assumed that the main contribution arises from the value at which the cutoff functions vanish. Thus the set of equations reduces to a parametric set instead of a continuous function.

$$
\begin{aligned}
\frac{\partial \tilde{g}_{s t}^{n m}(\omega)}{\partial \ln \Lambda} & =-\sum_{\nu}\left(g_{t t}^{\nu m}\left(\mu_{\nu}\right) \tilde{g}_{s t}^{n \nu}\left(\mu_{\nu}+K / 2\right) \Theta_{\omega-\mu_{\nu}-K / 2}\right. \\
& \left.+g_{t t}^{n \nu}\left(\mu_{\nu}\right) \tilde{g}_{s t}^{\nu m}\left(\mu_{\nu}-K / 2\right) \Theta_{\omega-\mu_{\nu}+K / 2}\right), \quad(77) \\
\frac{\partial g_{t t}^{n m}(\omega)}{\partial \ln \Lambda} & =-\sum_{\nu}\left(2 \tilde{g}_{s t}^{\nu m}\left(\mu_{\nu}-K / 2\right) \tilde{g}_{s t}^{n \nu}\left(\mu_{\nu}-K / 2\right) \Theta_{\omega-\mu_{\nu}+K}\right. \\
& +2 \tilde{g}_{s t}^{n \nu}\left(\mu_{\nu}+K / 2\right) \tilde{g}_{s t}^{\nu m}\left(\mu_{\nu}+K / 2\right) \Theta_{\omega-\mu_{\nu}-K} \\
& \left.+g_{t t}^{\nu m}\left(\mu_{\nu}\right) g_{t t}^{n \nu}\left(\mu_{\nu}\right) \Theta_{\omega-\mu_{\nu}}\right)
\end{aligned}
$$

We leave out this step while comparing the expressions (75) and (76) directly with Eqs. (56) and (57) derived with the flow equation method. However, the latter approximation is used in the numerical routines to accelerate the calculation.

\section{COMPARISON OF THE TWO METHODS TO ONE-LOOP ORDER}

In the following we will show that the two methods use the same approximations and therefore are identical to leading logarithmic order.

It is straightforward to see that the couplings in the two different calculations are related by $g_{t t}(\omega) \sim g_{k}^{\text {sum }}$ and $\tilde{g}_{s t}(\omega) \sim p_{k}$, where the energy $\epsilon_{k}$ is to be identified with the frequency (energy) $\omega(\hbar \omega)$.

We rewrite Eq. (56) and Eq. (76) to study in detail the commons and differences of the pRG and the flow 
equation method.

$$
\begin{aligned}
\frac{\partial g_{t t}^{n m}(\omega)}{\partial \ln \Lambda} & =-\sum_{\nu}\left(g_{t t}^{n \nu}(\omega) g_{t t}^{\nu m}(\omega) \Theta_{\omega-\mu_{\nu}}\right. \\
& +2 \tilde{g}_{s t}^{n \nu}(\omega+K / 2) \tilde{g}_{s t}^{\nu m}(\omega+K / 2) \Theta_{\omega+K-\mu_{\nu}} \\
& \left.+2 \tilde{g}_{s t}^{n \nu}(\omega-K / 2) \tilde{g}_{s t}^{\nu m}(\omega-K / 2) \Theta_{\omega-K-\mu_{\nu}}\right)
\end{aligned}
$$

$$
\begin{aligned}
\frac{d g_{k}^{\text {sum }, j}(B)}{d \ln \left(B^{-1 / 2}\right)}= & -\frac{1}{2} \sum_{\nu}\left(\left(g_{k}^{\text {sum }, j}\right)^{2} \mathrm{e}^{-2 B\left(\epsilon_{k}-\mu_{\nu}\right)^{2}}\right. \\
& +2\left(p_{k+K / 2}^{j}\right)^{2} \mathrm{e}^{-2 B\left(\epsilon_{k}+K-\mu_{\nu}\right)^{2}} \\
& \left.+2\left(p_{k-K / 2}^{j}\right)^{2} \mathrm{e}^{-2 B\left(\epsilon_{k}-K-\mu_{\nu}\right)^{2}}\right)
\end{aligned}
$$

The flow parameter $B$ is related to the frequency cutoff by $B=\Lambda^{-2}$ and $\mu_{v}= \pm V_{j} / 2$. Therefore the equations are actually identical down to the prefactors. The prefactor $1 / 2$ in Eq. (80) stems from the even-odd combination of the leads where $g^{\text {even }}=(1+r) g^{n m}$ and thus $g^{\text {even }}=2 g^{n m}$ for $r=1$.

Let us repeat the common approximation before we embark on the differences. Both methods take into account only the leading logarithmic order by treating only the lowest order diagrams which contribute to the scaling. In the perturbative RG the pseudoparticle energies are taken to be onshell which corresponds to treating the spin operators without a bath. In both methods the level of complexity is reduced by imposing energy conservation on the vertex, i.e. the energy of the outgoing conduction electron is given by the energy of the incoming electron diminished by eventual inelastic processes inside the dot. Since both methods keep information on the whole bandwidth and the renormalization reduces only the bandwidth of the state that one scatters into, they are destined to treat non-equilibrium physics on different energy scales, i.e. when physics of more than the ground state play a role.

The main difference are the two different cutoff functions $e^{-B x^{2}}=e^{-(x / \Lambda)^{2}}$ versus $\Theta_{x}=\Theta(\Lambda-|x|)$. The step function is an approximation in the perturbative $R G$ whereas the exponential cutoff arises naturally in the flow equation method approach. The form of the cutoff influences at most the lineshape in the proximity of logarithmically divergent couplings, see Fig. 3 and 4 . Since these are heavily influenced by higher-order renormalization, their shape in leading logarithmic order is not reliable anyway and only the flow at the corresponding energies is described correctly. Note though, that for example in the current or other physical observables, it is the average over a voltage window which enters, i.e. the integral over the frequency-dependent couplings. Therefore this difference can play a role when comparing result for the non-equilibrium current.

Furthermore there is a summation over the lead indices in the perturbative RG. In the flow equation calculation we used the symmetry that only the even channel of each lead is coupled to the dot and therefore the lead index drops out. In general the model can also be studied without doing the even-odd transformation ${ }^{7}$, e.g. to study models which are not equivalent to the Anderson impurity model by the Schrieffer-Wolff transformation. As explained in the introductory part, e.g. the important transport part $g^{12}$ between two leads on the left side is given by the asymmetry parameter and $g^{L / R, L}=\left(1+r_{L}\right) g^{L / R ; 22}: g^{L / R ; 12}=\sqrt{r_{L}} /\left(1+r_{L}\right) g^{L / R, L}$. We concentrate for the comparison only on the symmetric case $r_{L}=1$ and consequently find that $g_{k}^{\text {sum }}=2 g_{t t}(\omega)$ and $p_{k}=2 \tilde{g}_{s t}(\omega)$.

The strength of the flow equation method is that the diagonal parametrization is a convenient choice in order to find analytical expressions, but not a necessary limitation of the method. In practice the numerical cost limits the evaluation of the flow equations to the diagonal parametrization.

Nevertheless both methods are still bound to break down due to the strong-coupling behavior of the Kondo correlations. In the following we will explain how decoherence effects are included in the two different methods, how they provide an additional cutoff and in the end compare the two methods again.

\section{BEYOND ONE-LOOP}

\section{A. In perturbative RG}

The Kondo problem at zero temperature is known not to be solvable by perturbation theory. Even though the renormalization schemes can improve the limit of validity due to an appropriate summation of diagrams, the theory still breaks down at the energy scale $\Lambda=T_{K}$. However, in equilibrium at finite temperature $T \gtrsim T_{K}$ or in nonequilibrium for sufficiently large voltage bias one expects a well-behaved weak coupling expansion.

The seminal work of A. Rosch et al. 2 on the nonequilibrium Kondo model achieves this by including a physically motivated cutoff given by the current-induced noise in the system. Decoherence is unavoidably present due to the non-zero steady current flow through the system, which generates Johnson-Nyquist current noise. In other words, the quantum dot states gain a finite lifetime due to elastic or inelastic cotunneling processes with the leads. Also decay rates due to external baths can play a role. In general a non-zero decoherence rate $\Gamma$ has to be included in the retarded Green's function. This leads to a new scaling behavior since the leading logarithmic diagrams changes as

$$
\begin{aligned}
& \Lambda \frac{\partial}{\partial \Lambda} \int_{-\Lambda}^{\Lambda} d \epsilon \frac{x-\epsilon}{(x-\epsilon)^{2}+\Gamma^{2}} \tanh \left(\frac{\epsilon}{2 T}\right) \\
& \approx-\Lambda \frac{x-\Lambda}{(x-\Lambda)^{2}+\Gamma^{2}}+\Lambda \frac{x+\Lambda}{(x+\Lambda)^{2}+\Gamma^{2}} .
\end{aligned}
$$


Thus the cutoff has to be corrected to be

$$
\Theta_{x}=\Theta\left(\Lambda-\sqrt{x^{2}+\Gamma^{2}}\right)
$$

instead of just $\Theta(\Lambda-|x|)$. It was shown perturbatively in Ref. 13 that in non-equilibrium selfenergy and vertex correction become important to the same degree. Further studies using different renormalization group methods confirmed the observation that the non-equilibrium decoherence rate are determined by transport processes and can be different to the thermodynamically expected expressions,$\underline{4,5,24-26}$

The statement that decoherence terms have to be included is equivalent to the failure of the on-shell assumption since the levels in the quantum dot are broadened and thus the spin state on the quantum dot gains a finite lifetime. To find the correct cutoff one has to calculate the spin susceptibility and find the correct Lorentzian shape.

In Fig. 3] we show the flow of the triplet-triplet coupling $g_{t t}^{n m}(\omega)$ for $n=m=1$ as a function of the frequency $\omega$ and for different values of the cutoff $\Lambda$.

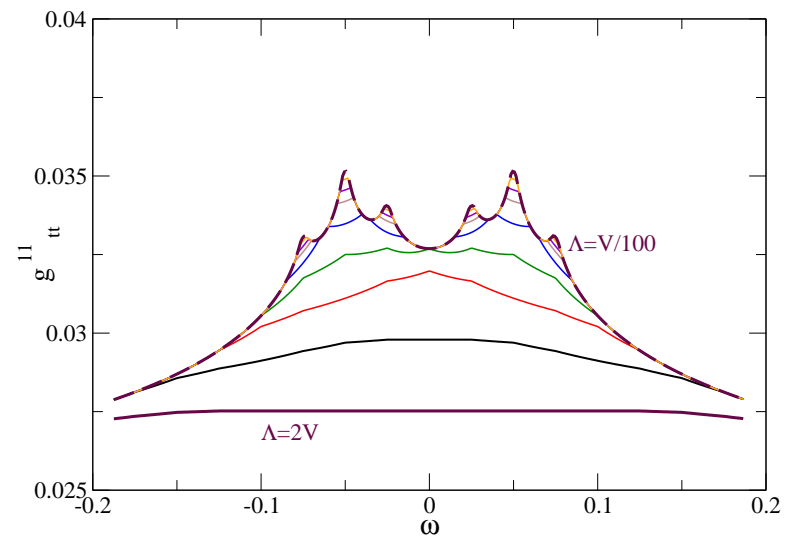

FIG. 3: Poor man's scaling including a frequency dependence of the coupling for a Double Quantum Dot system; $g_{t t}^{11}=0.025, N(0) K=0.025, T \approx 0$, $N(0)\left(e V_{L}\right) \equiv V=0.1, N(0)\left(e V_{R}\right)=0$; values of the cutoff $\Lambda / V=2,1,1 / 2,1 / 5,1 / 10,1 / 15,1 / 25,1 / 50,1 / 100$ where the first (solid line) and the last value (dashed line) are marked extra in the plot.

For a large cutoff $\Lambda$ the coupling is a constant and does not depend on the frequency of the scattered electrons. As the cutoff is reduced a frequency dependence evolves and the coupling continues to grow at the resonant energy scales, i.e. at $\omega= \pm V / 2, \pm K \pm V / 2$, while the flow does not continue at other energy scales due to $\Theta_{\omega}$. Once the energy scale which is needed for a scattering process is smaller than the bandwidth, no further processes can be integrated out and it is expected that the flow should not continue. Including the frequency dependence and the frequency cutoff $\Theta_{\omega}$ in the poor man's scaling equations produces this behavior automatically, which is one of the advantages of the pRG method.

Without the cutoff given by $\Gamma$ the couplings at the Fermi energies $\omega= \pm V / 2$ are logarithmically divergent and the poor man's scaling approach breaks down. Including $\Gamma$ in the cutoff function $\Theta_{\Gamma}$ simulates the physical observation that similar to a non-zero temperature $T \neq 0$ the infinite series of infinitesimal spin-flip excitations around the Fermi edge is interrupted by a noncoherent process. The flow of the coupling stops at $g_{t t} \propto 1 / \ln \left(\Gamma / T_{K}\right)^{14}$ assuming that $\Gamma$ is the relevant cutoff (which can otherwise also be proportional to $T$ or combinations of internal energies).

Note that the divergence of the coupling at the Fermi edge is not necessarily equivalent to the breakdown of the calculation. For example in the calculation of the non-equilibrium current the average of the frequencydependent coupling over the whole voltage window needs to be included where the divergent coupling is just a boundary term ${ }^{26}$.

\section{B. In the flow equation method}

In the flow equation method, the generalization to higher orders in the coupling function is straightforward as has already been illustrated in Refs. 6 - 8 .

In second order in the Kondo coupling $J$ new interaction terms are created. These can be compared to a 6 -leg vertices with two incoming and two outgoing conduction electrons and one incoming and one outgoing spin state of the quantum dot in the pseudo-fermion language. Contributions to the flow of the four-leg Kondo interaction vertices are generated by a product of one of the newly generated vertices with the initial Kondo vertex. Note that since two conduction electron lines are thus integrated out, these terms are very similar to the self energy and vertex corrections discussed in the previous section.

In contrast to the one-loop contribution, the two-loop contribution includes an integration over two intermediate energies, i.e. an electron-hole pair like $n(q v)(1-$ $\left.n\left(q^{\prime} v\right)\right)$. This integration is taken over a window of energies, e.g. voltage window or singlet-triplet transition window. Besides the standard two-loop term in the $\beta$-function $d g / d \ln \Lambda=-2 g^{2}+2 g^{3}$ for a spin- 1 we also expect to find contributions from $g^{3} V / \Lambda$ or $g^{3} K / \Lambda$, i.e. coupling ${ }^{3} \times$ phase space. These arise naturally in the calculation and can be identified as the cutoff rates given by e.g. the Johnson-Nyquist current noise.

\section{Discussion of the flow to second order}

In the case of a double quantum dot system these newly generated couplings are 


$$
\begin{aligned}
H_{i n t}^{(2)}= & \sum_{j v} \sum_{k^{\prime} k ; q^{\prime} q} K_{k^{\prime} k ; q^{\prime} q}^{\text {sum }} i:\left(\vec{S}_{L}+\vec{S}_{R}\right)\left(\vec{s}_{\left(k^{\prime} j\right)(k j)} \times \vec{s}_{\left(q^{\prime} v\right)(q v)}\right): \\
& +\sum_{j v} \sum_{k^{\prime} k ; q^{\prime} q} K_{k^{\prime} k ; q^{\prime} q^{\prime}}^{P} i:\left(\left(\vec{S}_{L}-\vec{S}_{R}\right)+2 i\left(\vec{S}_{L} \times \vec{S}_{R}\right)\right)\left(\vec{s}_{\left(k^{\prime} j\right)(k j)} \times \vec{s}_{\left(q^{\prime} v\right)(q v)}\right): \\
& +\sum_{j v} \sum_{k^{\prime} k ; q^{\prime} q} K_{k^{\prime} k ; q^{\prime} q}^{M} i:\left(\left(\vec{S}_{L}-\vec{S}_{R}\right)-2 i\left(\vec{S}_{L} \times \vec{S}_{R}\right)\right)\left(\vec{s}_{\left(k^{\prime} j\right)(k j)} \times \vec{s}_{\left(q^{\prime} v\right)(q v)}\right): \\
& +\sum_{j v} \sum_{k^{\prime} k ; q^{\prime} q} K_{k^{\prime} k ; q^{\prime} q}^{R K K Y} i 2 i\left(:\left(\vec{S}_{L} \times \vec{s}_{\left(k^{\prime} j\right)(k j)}\right)\left(\vec{S}_{R} \times \vec{s}_{\left(q^{\prime} v\right)(q v)}\right)+\left(\vec{S}_{R} \times \vec{s}_{\left(k^{\prime} j\right)(k j)}\right)\left(\vec{S}_{L} \times \vec{s}_{\left(q^{\prime} v\right)(q v)}\right):\right)
\end{aligned}
$$

These interactions are not present in the initial flow

$$
\begin{gathered}
K_{k^{\prime} k ; q^{\prime} q}^{\text {sum }}(B=0)=0 \\
K_{k^{\prime} k ; q^{\prime} q}^{P}(B=0)=0 \\
K_{k^{\prime} k ; q^{\prime} q}^{M}(B=0)=0 \\
K_{k^{\prime} k ; q^{\prime} q}^{R K K}(B=0)=0
\end{gathered}
$$

They can be interpreted as an entanglement of the quantum dot states with the spin states of the conduction electrons. This can be seen by studying of the flow of the coupling function where we find that the initial free spin state $\left(\vec{S}_{L}+\vec{S}_{R}\right)$ evolves into the entangled spin state $\left(\vec{S}_{L}+\vec{S}_{R}\right) \times \vec{s}_{\left(q^{\prime} v\right)(q v)}$ for $B \rightarrow \infty$. Correspondingly we observe in the flow of the Hamiltonian that the coupling $J_{k^{\prime} k}$ starts to decrease at an energy scale when $K_{k^{\prime} k ; q^{\prime} q}$ starts to grow. The flow of $J_{k^{\prime} k}$ is reversed in the sense that it does not diverge logarithmically but for $B \rightarrow \infty$ we observe $J_{k^{\prime} k} \rightarrow 0$ while the values of the couplings in $H_{\text {int }}^{(2)}$ grow.

Note that also an RKKY-like interaction is created to this order. All kinds of potential scattering contributions are neglected here, similar to the pRG calculation, because they do not contribute in the wide band limit. Since we study only antiferromagnetic coupling between the two spins we can neglect the RKKY interaction in the following. For a discussion of the flow of this coupling we refer the interested reader to appendix B where it is shown that the coupling $K$ is similar to a magnetic field in a spin- $1 / 2$ Kondo problem renormalized by the

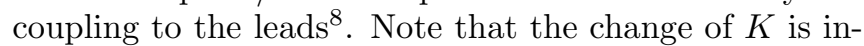
cluded in the numerical results shown here if not stated otherwise.

The rather complicated entanglement term

$$
\begin{aligned}
& K_{k^{\prime} k ; q^{\prime} q}^{R K K} i 2 i\left(:\left(\vec{S}_{L} \times \vec{s}_{\left(k^{\prime} j\right)(k j)}\right)\left(\vec{S}_{R} \times \vec{s}_{\left(q^{\prime} v\right)(q v)}\right)\right. \\
& \left.+\left(\vec{S}_{R} \times \vec{s}_{\left(k^{\prime} j\right)(k j)}\right)\left(\vec{S}_{L} \times \vec{s}_{\left(q^{\prime} v\right)(q v)}\right):\right) \\
& =(-4) K_{k^{\prime} k ; q^{\prime} q}^{R K K Y}:\left(\vec{S}_{L} \vec{S}_{R}\right)\left(\vec{s}_{\left(k^{\prime} j\right)(k j)} \vec{s}_{\left(q^{\prime} v\right)(q v)}\right): \\
& \quad+2 K_{k^{\prime} k ; q^{\prime} q}^{R K Y}:\left(\vec{S}_{L} \vec{s}_{\left(k^{\prime} j\right)(k j)}\right)\left(\vec{S}_{R} \vec{s}_{\left(q^{\prime} v\right)(q v)}\right): \\
& +2 K_{k^{\prime} k ; q^{\prime} q}^{R K K Y}:\left(\vec{S}_{R} \vec{s}_{\left(k^{\prime} j\right)(k j)}\right)\left(\vec{S}_{L} \vec{s}_{\left(q^{\prime} v\right)(q v)}\right):
\end{aligned}
$$

reproduces an RKKY-like interaction. Note that during the flow $\vec{S}_{L} \vec{S}_{R} \rightarrow\left(\vec{S}_{L} \times \vec{s}_{\left(k^{\prime} j\right)(k j)}\right)\left(\vec{S}_{R} \times \vec{s}_{\left(q^{\prime} v\right)(q v)}\right)$ and therefore the additional term is effectively a rescaled exchange interaction. This term is not present for example in the spin- $1 / 2$ model and special to the exchange coupled quantum dot.

We now study systematically how the new terms in the Hamiltonian decompose into the initial coupling terms. A detailed expression can be found in the appendix C. Here we write down only the final expression for the symmetric setup discussed throughout the paper. The flow of the coupling $g_{k}^{\text {sum, } j}(B)$ in diagonal parametrization yields

$$
\begin{aligned}
\frac{d g_{k}^{\text {sum }, j}(B)}{d B} & =\frac{1}{2 B} \frac{1}{2} \sum_{v= \pm} \mathrm{e}^{-2 B\left(\epsilon_{k}+v V_{j} / 2\right)^{2}}\left(g_{k}^{\text {sum }, j}\right)^{2} \\
& +2 \frac{1}{2 B} \frac{1}{2} \sum_{v= \pm} \mathrm{e}^{-2 B\left(\epsilon_{k}+v V_{j} / 2+K\right)^{2}}\left(p_{k+K / 2}^{j}\right)^{2} \\
& +2 \frac{1}{2 B} \frac{1}{2} \sum_{v= \pm} \mathrm{e}^{-2 B\left(\epsilon_{k}+v V_{j} / 2-K\right)^{2}}\left(p_{k-K / 2}^{j}\right)^{2} \\
& -\frac{1}{4 B} \sum_{v} g_{k}^{\text {sum }, j}(B)\left(g_{\text {max }}^{\text {sum }, v}(B)\right)^{2} \\
& -\sum_{v} f\left(0, V_{v}\right) g_{k}^{\text {sum }, j}(B)\left(g_{\text {max }}^{\text {sum }}(B)\right)^{2} \\
- & \left(\frac{1}{4 B} \mathrm{e}^{-2 B K^{2}}+\frac{K}{4} \sqrt{\frac{\pi}{2 B}} \operatorname{erf}(\sqrt{2 B} K)\right) \\
& \times \sum_{v} g_{k}^{\text {sum }, j}(B)\left(2 p_{\text {max }}^{v}(B)\right)^{2} \\
- & \sum_{v} f\left(K, V_{v}\right) g_{k}^{\text {sum }, j}(B)\left(2 p_{\text {max }}^{v}(B)\right)^{2}
\end{aligned}
$$


and

$$
\begin{aligned}
\frac{d p_{k}^{j}(B)}{d B}= & \frac{1}{2 B} \frac{1}{2} \sum_{v= \pm} \mathrm{e}^{-2 B\left(\epsilon_{k}+v V_{j} / 2-K / 2\right)^{2}} g_{k-K / 2}^{\text {sum }, j} p_{k}^{j} \\
& +\frac{1}{2 B} \frac{1}{2} \sum_{v= \pm} \mathrm{e}^{-2 B\left(\epsilon_{k}+v V_{j} / 2+K / 2\right)^{2}} p_{k}^{j} g_{k+K / 2}^{\text {sum }, j} \\
& -\frac{1}{4 B} \sum_{v} p_{k}^{j}(B)\left(g_{\max }^{\text {sum }, v}(B)\right)^{2} \\
& -\sum_{v} f\left(0, V_{v}\right) p_{k}^{j}(B)\left(g_{\max }^{\text {sum }, v}(B)\right)^{2} \\
& -\left(\frac{1}{4 B} \mathrm{e}^{-2 B K^{2}}+\frac{K}{4} \sqrt{\frac{\pi}{2 B}} \operatorname{erf}(\sqrt{2 B} K)\right) \\
& \times \sum_{v} p_{k}^{j}(B)\left(2 p_{\max }^{v}(B)\right)^{2} \\
& -\sum_{v} f\left(K, V_{v}\right) p_{k}^{j}(B)\left(2 p_{\max }^{v}(B)\right)^{2}
\end{aligned}
$$

where for $r_{L}=r_{R}=1$

$$
\begin{aligned}
f\left(K, V_{v}\right)= & \frac{1}{4 B} \frac{1}{4}\left(\mathrm{e}^{-2 B\left(K-V_{v}\right)^{2}}+\mathrm{e}^{-2 B\left(K+V_{v}\right)^{2}}-2 \mathrm{e}^{-2 B K^{2}}\right) \\
+\frac{1}{4} & \sqrt{\frac{\pi}{2 B}} \frac{1}{4}\left[\left(K-V_{v}\right) \operatorname{erf}\left(\sqrt{2 B}\left(K-V_{v}\right)\right)\right. \\
& +\left(K+V_{v}\right) \operatorname{erf}\left(\sqrt{2 B}\left(K+V_{v}\right)\right) \\
& -2 K \operatorname{erf}(\sqrt{2 B} K)]
\end{aligned}
$$

and in particular

$$
\begin{aligned}
f\left(0, V_{v}\right) & =\frac{1}{4 B} \frac{1}{2}\left(\mathrm{e}^{-2 B V_{v}^{2}}-1\right) \\
& +\frac{1}{8} \sqrt{\frac{\pi}{2 B}} V_{v} \operatorname{erf}\left(\sqrt{2 B}\left(V_{v}\right)\right) .
\end{aligned}
$$

Note that we assumed that the frequency dependence is given dominantly by the exponential decay and therefore the couplings in the integration over the energy window can be approximated by their most divergent term, i.e. $g_{\left(q^{\prime}+q\right) / 2}^{\mathrm{sum}, v} \approx g_{\max }^{\mathrm{sum}, v}=g_{0}^{\mathrm{sum}, v}$ if voltage $V_{v}$ is zero. For $V_{v}=0$ there is no contribution from $f(0,0)=f(K, 0)=0$. On the contrary for finite voltage and $B \gg V_{v}^{-2}: f\left(0, V_{v}\right) \approx \frac{1}{2 B}+\frac{V_{v}}{2} \sqrt{\frac{\pi}{2 B}} \rightarrow \frac{V_{v}}{2} \sqrt{\frac{\pi}{2 B}}$ since $\sqrt{\frac{\pi}{2 B}} \gg \frac{1}{2 B}$ and $f\left(0, V_{v}\right)$ provides the leading contribution to the flow.

\section{Two-loop results of the Double Quantum Dot system}

In the equilibrium case, where the applied voltages $V_{L}=V_{R}=0$, the scaling equation for $g_{k}^{\text {sum }}$ is given by

$$
\begin{aligned}
\frac{d g_{k}^{\text {sum }, j}(B)}{d B} \approx & \frac{1}{2 B} \mathrm{e}^{-2 B \epsilon_{k}^{2}}\left(g_{k}^{\text {sum }, j}\right)^{2} \\
+ & 2 \frac{1}{2 B} \mathrm{e}^{-2 B\left(\epsilon_{k}+K\right)^{2}}\left(p_{k+K / 2}^{j}\right)^{2} \\
+ & 2 \frac{1}{2 B} \mathrm{e}^{-2 B\left(\epsilon_{k}-K\right)^{2}}\left(p_{k-K / 2}^{j}\right)^{2} \\
- & \frac{1}{4 B} \sum_{v} g_{k}^{\text {sum }, j}(B)\left(g_{\text {max }}^{\text {sum }}(B)\right)^{2} \\
- & \left(\frac{1}{4 B} \mathrm{e}^{-2 B K^{2}}+\frac{K}{4} \sqrt{\frac{\pi}{2 B}} \operatorname{erf}(\sqrt{2 B} K)\right) \\
& \quad \times \sum_{v} g_{k}^{\text {sum }, j}(B)\left(2 p_{\text {max }}^{v}(B)\right)^{2} .
\end{aligned}
$$

Note that the scaling equation is of the form of a twoloop calculation with an additional contribution proportional to $(K / \sqrt{2 B}) \operatorname{erf}(\sqrt{2 B} K)$. For large values of the argument in the error function we can approximate $\operatorname{erf}(x) \rightarrow \operatorname{sign}(x)$. Therefore we find in the limit $B \rightarrow \infty$ that this term is $\propto 1 / \sqrt{B}>1 / B$ and thus this term dominates the asymptotic flow. As discussed later on, this provides a cutoff in the flow given by the singlettriplet exchange interaction $K$. This cutoff can prevent the divergence of the elastic Kondo coupling $g_{k}^{\text {sum }}$ by favoring a singlet formation of the two dots instead of a Kondo singlet with the leads if $K$ is larger than a critical value. This is a signature of the quantum phase transition in our system and will be discussed in more detail in another publication 27 .

Furthermore we try to illustrate the effect of these decoherence terms in the two-loop calculation in the limit $V_{j} \gg K$ and refer the reader to the explicit discussions in the literature to non-equilibrium flow equations for further reading 17 .

Note that we have to distinguish between the voltage applied on the left side or right side since there is a summation over the left and right lead index in third order in the coupling. For now we will assume $V_{R}=0$ and only $V_{L} \equiv V \neq 0$. In the current this can lead to the interesting effect of a transconductance ${ }^{14}$ as discussed at the end of this section.

For $2 B V^{2} \gg 1 \gg 2 B K^{2}$ such that we can set $\operatorname{erf}(\sqrt{2 B} V) \approx 1$ and neglect contributions from $\mathrm{e}^{-2 B K^{2}}$ we find the scaling equation

$$
\begin{aligned}
\frac{d g_{k}^{\text {sum }, j}(B)}{d B} & \approx \frac{1}{2 B} \sum_{\alpha= \pm 1} \frac{1}{2} \mathrm{e}^{-2 B\left(\epsilon_{k}+\alpha V / 2\right)^{2}}\left(g_{k}^{\text {sum }, j}\right)^{2} \\
& -\sum_{v=L, R}\left[\frac{1}{4} \frac{V_{v}}{2} \sqrt{\frac{\pi}{2 B}}\right] g_{k}^{\text {sum }, j}(B)\left(g_{\text {max }}^{\text {sum }, v}(B)\right)^{2} \\
& -\sum_{v=L, R}\left[\frac{1}{4} \frac{V_{v}}{2} \sqrt{\frac{\pi}{2 B}}\right] g_{k}^{\text {sum }, j}(B)\left(p_{\text {max }}^{v}(B)\right)^{2}
\end{aligned}
$$

In this limit the voltage $V$ dominates the flow for $B \rightarrow \infty$ and thus provides a cutoff scale for the flow. 


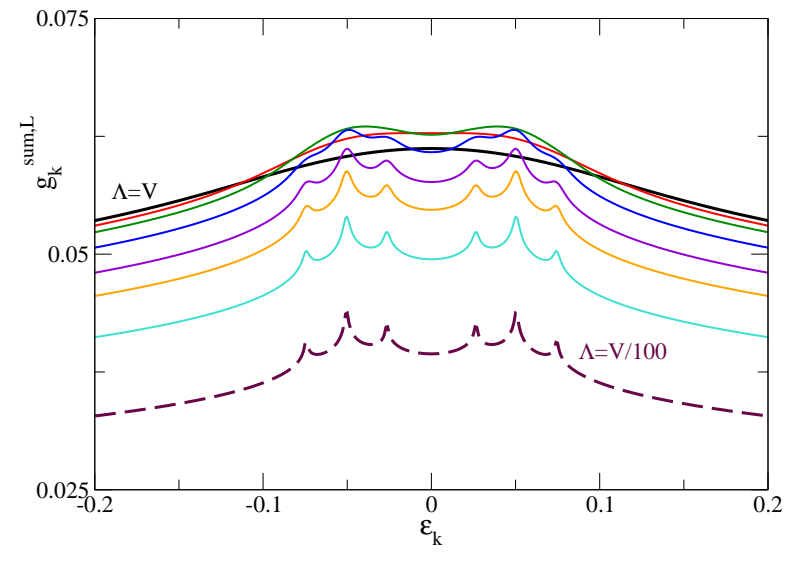

FIG. 4: Flow of $g_{k}^{\text {sum }, L}$ as a function of energy $\epsilon_{k}$ for different values of the flow parameter $B=\left(1 / \Lambda^{2}\right)$ where $\Lambda$ chosen identical to Fig. 3 Furthermore $N(0) K=0.025 N(0)(e V)=$ $0.01, T \approx 0$ and $g\left(B_{0}\right)=0.05$. Same parameters as in Fig. 3

The actual decoherence rate has to be determined studying the spin susceptibility of the system. Nevertheless, it has been shown e.g. for the spin-1/2 Kondo model ${ }^{7}$ that the rate $\Gamma$ read off from the correlation function is equivalent to the observed cutoff in the scaling equation.

In Fig. 4 we show (similar to Fig. 3) the flow of the coupling $g_{k}^{\text {sum,j }}$ as a function of $\epsilon_{k}$ for different values of the flow parameter $B$. As in the pRG calculation the coupling is initially equal for all values of the energy and a frequency dependence evolves slowly with increasing values of $B$. Note the difference between the two calculations: in the flow equation method the two-loop contribution leads to a decrease in the running couplings as soon as the energy scale $\Gamma$ is reached. At this stage the higher order coupling terms in $H_{i n t}^{(2)}$ start to grow and the entangled spin state determines the physics. This is seen as a decrease in the initial Kondo coupling $g_{k}^{\mathrm{sum}, j}$, whereas in the poor man's scaling approach the flow is just stopped at this scale and then stays constant.

It is still an open issue if the coupling exactly at $B=1 / \Gamma^{2}$ can be used if physical observables are calculated, and if the cutoff scheme motivated by a self energy cutoff is valid to higher orders. For the parameter set chosen in Fig. 4 the peak structure only evolves after the absolute value of the coupling goes down. Otherwise the overall behavior is very similar to the result using $\mathrm{pRG}$ as shown in Fig. 3. A direct comparison of results of the two methods can be found in the next subsection.

\section{Transconductance}

A non-zero transconductance is found in the double quantum dot system ${ }^{14}$. If the ground state of the double quantum dot system is given by the degenerate triplet states $(K<0)$, we find a zero-bias resonance which can be Kondo enhanced. On the other hand if the double quantum dot system is in the non-degenerate singlet ground state $(K>0)$, transport is blocked for voltages below a threshold given by the exchange interaction $K$. Once the applied voltage is larger than $K$, transport is also allowed which includes the three triplet states and we find an inelastic cotunneling step which is logarithmically enhanced by the Kondo correlations. A non-equilibrium occupation of the triplet becomes possible due to the large applied voltage. For example for a large voltage applied on the left side the linear response current on the right side is not blocked but there is a non-zero signal. This transconductance $d I_{R} / d V_{L}$ was studied in detail by one of the authors 14,15 .

The transfer of decoherence from one quantum dot to another can also be seen in the flow equation method. In contrast to one-loop order where only one virtual process is allowed, there are two intermediate states which contribute to two-loop order. Note that there is a summation over the lead index in Eq. (90). Therefore an electron-hole pair created in e.g. the right lead due to a finite voltage enters the scaling equation for the coupling to the left leads.

The signatures of the transconductance can thus be observed as a cutoff of the divergent coupling to the right lead even when the voltage is only applied to the left lead, see Fig. 5] Note that the decoherence scale though

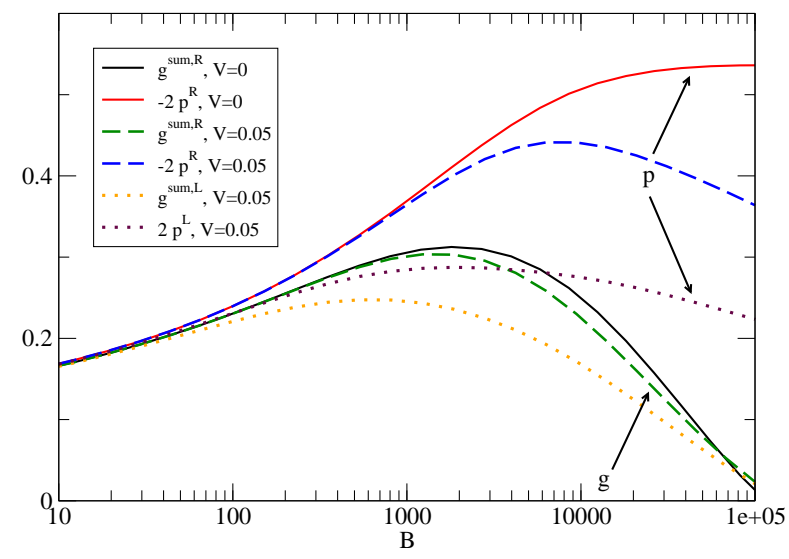

FIG. 5: Flow equation solution using the mean value of the couplings $g_{\text {mean }}^{\mathrm{sum}, R}$ and $-2 p_{\text {mean }}^{\mathrm{sum}, R}$ to the right leads; solid lines illustrate the flow for voltage $V_{L}=V_{R}=0$. In this case the singlet-triplet excitation energy $K$ provides a cutoff scale in $g_{\text {mean }}^{\text {sum }}$. For a finite voltage applied to the left lead, i.e. $T_{K}=$ $0.005, N(0) K=0.025, V_{R}=0$ and $N(0)\left(e V_{L}\right) \equiv V=0.05$, we show again $g_{\text {mean }}^{\text {sum, } R}$ and $-2 p_{\text {mean }}^{\text {sum, } R}$ (dashed lines). A decay of the couplings sets in which is initiated by the decoherence on the left side of the DQD. See discussion of the transconductance effect in the text. For comparison we also show that the decoherence rate for $g_{\text {mean }}^{\mathrm{sum}, L}$ and $2 p_{\text {mean }}^{\mathrm{sum}, L}$ (dotted lines) is larger.

is larger than the decoherence on the right side. To study the transition from a strong-coupling to a weak-coupling problem, the calculation of either the transconductance or another physical quantity is necessary. This is not the focus of this paper. Here we concentrate on a comparison between the flow equation method and pRG scaling. We 
have shown that they are identical to lowest order and by Fig. [5 also that the flow equation method provides the same physics as was found from previous studies of the double quantum dot system using $\mathrm{pRG}^{14}$.

\section{Comparison}

As a further comparison we show the mean values of the couplings in the two different calculations. The aver-

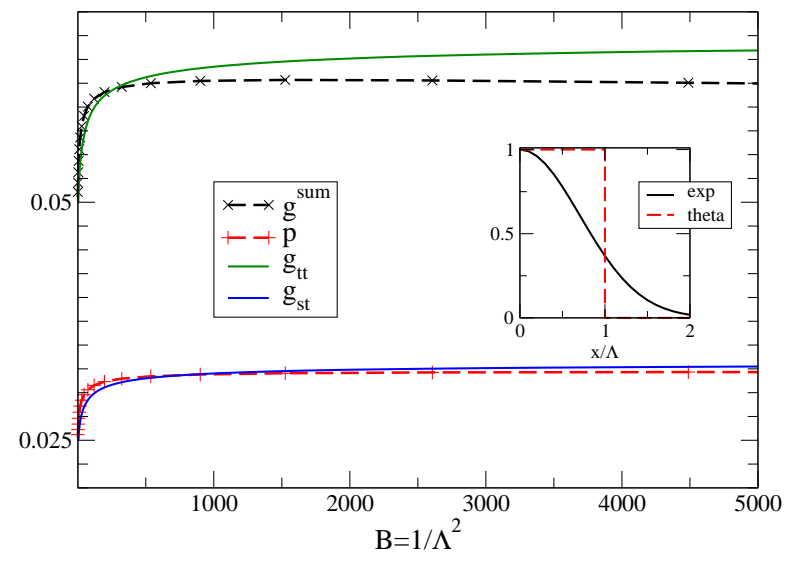

FIG. 6: Comparison of the averaged coupling functions in pRG (solid lines: $g_{t t} \hat{=} 2\left[g_{t t}^{11}\right]_{\text {mean }}$ and $g_{s t} \hat{=} 2\left[\tilde{g}_{s t}^{11}\right]_{\text {mean }}$ ) and flow (dashed lines: $g^{\text {sum }} \hat{=} g_{\text {mean }}^{\text {sum }, L}$ and $p \hat{=} p_{\text {mean }}^{L}$ ), same parameters as in Fig. 3 and 4 Inset: comparison of the different cutoff functions, $\theta_{x}$ and $\exp \left(-(x / \Lambda)^{2}\right)$

aged quantities enter physical quantities like the current and spin susceptibilities. They are defined as

$$
\begin{aligned}
& g_{\text {mean }}^{\text {sum }, j}=\frac{1}{V_{j}+2 K} \int_{-V_{j} / 2-K}^{V_{j} / 2+K} d \epsilon_{k} g_{k}^{\text {sum }, j} \\
& p_{\text {mean }}^{j}=\frac{1}{V_{j}+K} \int_{-V_{j} / 2-K / 2}^{V_{j} / 2+K / 2} d \epsilon_{k} p_{k}^{j}
\end{aligned}
$$

and analogous for the mean values of $g_{t t}^{n m}(\omega)$ and $\tilde{g}_{s t}^{n m}(\omega)$.

In Fig. 6 we show a comparison of the triplet-triplet and singlet-triplet couplings versus the cutoff/flow parameter for the same set of parameters as discussed before. We observe that the couplings in both methods start to grow logarithmically. The quantitative behavior is slightly different due to the different cutoff schemes. Note that the plotted variable is the averaged value over a frequency/energy regime which thus includes the slopes of the most divergent coherent couplings, compare Fig. 3 and 4. These are different due to either a cutoff of $\mathrm{e}^{-(x / \Lambda)^{2}}$ or $\Theta(\Lambda-x)$ as illustrated in the inset of Fig. [6. Both methods are limited to weak-coupling and thus the value of $g_{\text {mean }}^{\text {sum }}$ changes only by roughly $25 \%$ and stays well below the strong-coupling limit.

Note that we compare the two-loop flow equations with the decoherence-cutoff corrected pRG. The philosophies of the two different methods are obvious in Fig. 6. In the pRG the flow continues to grow logarithmically and as soon as the reduced band reaches the cutoff, the flow stops and the coupling stays constant. This is the value which is then inserted into the expression of physical observables like the current or the transconductance, etc. In the flow equation method the decoherence enters differently. As soon as the flow parameter $B$ reaches $1 / \Gamma^{2}$, the initial couplings start to decay again. Once the decoherence scale is reached the Hamiltonian changes its form, i.e. in the Kondo model the impurity spin is entangled with the leads and therefore the newly generated couplings in $H_{\text {int }}^{(2)}$ start to grow and determine the dynamics of the system. For $B \rightarrow \infty$ the Kondo coupling would thus flow to 0 and the physical relevant value of the Kondo coupling should be chosen as the maximum before the value starts to decay.

The two decoherence scales of the two different methods for the example in Fig. 6] seem very different since the pRG flow still continues while the couplings in the flow equation methods already start to decay. This observation can be traced back to two reasons. First, it is not obvious if $B=1 / \Lambda^{2}$ is identically fulfilled or if there is some prefactor involved which invalidates the direct comparison. Second, as pointed out before an additional cutoff is found in the flow equation method which is proportional to the exchange interaction $K$ and not given by the noise fluctuations. This term is not found from a Korringa-rate calculation in the pRG method. On the other hand the term contains the physics of the quantum phase transition in the double quantum dot system since the Kondo coupling is expected to diverge only if a Kondo singlet is built up with the conduction electrons in the leads, and not if the quantum dot is locked in a singlet configuration. Detailed study of the behavior of this transition is the subject of a future publication 27 .

The best comparison which is thus possible between the two methods is the frequency-dependent coupling directly at the decoherence scale in the flow equation method and for $\Lambda \rightarrow 0$ in the pRG approach. This is shown in Fig. 7. The parameters are chosen such that the voltage is the determining cutoff scale and are identical to the parameters in all other figures. Note that we only show the coupling in the lead where the voltage is applied in contrast to the discussion of the transconductance. We can conclude that the flow equation method and the pRG approach describe the same physics of decoherence contributions out of equilibrium.

\section{CONCLUSION AND OUTLOOK}

In conclusion we have shown for the example of the double quantum dot system that the flow equation method $\mathrm{g}^{\underline{6}}$ and the poor man's scaling approach to nonequilibrium ${ }^{2}$ are equivalent to one-loop order. In both methods we find that the coupling develops a frequency dependence and that only at energies/frequencies where coherent processes are possible a logarithmic divergence 


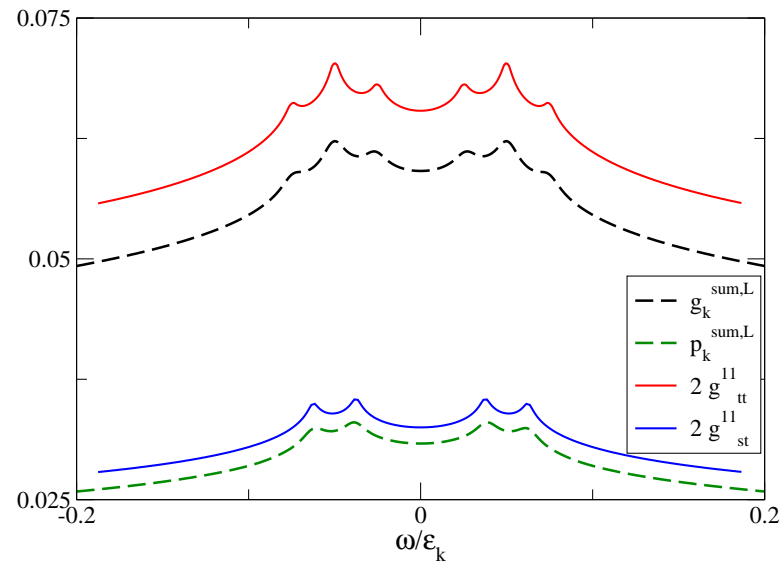

FIG. 7: Comparison of the coupling $g_{t t}^{11}(\omega)\left(\tilde{g}_{t s}^{11}(\omega)\right)$ in the pRG (solid lines) for $\Lambda \rightarrow 0$ and $g_{k}^{\text {sum, } L}\left(p_{k}^{L}\right)$ for the flow equation (dashed lines) at the decoherence scale $B=1 / \Gamma^{2}$. Parameters are chosen to be the same as in the previous figures. Note that we compare a 2-loop calculation (flow equation) to an effective theory which mimics the effects of 2-loop contributions.

of the coupling can be observed.

If the infinite series of coherent processes is broken by decoherence, e.g. like in the presence of a not negligible current, the divergence should be lifted. The two different methods use different approaches for this: while the pRG approach includes a physically motivated cutoff given by lifetime broadening and/or vertex corrections, the flow equation method is continued to higher order, i.e. two-loop, where a cutoff arises systematically.

The double quantum dot setup is interesting to study since it is the simplest model which includes spincoherent inelastic scattering processes like the singletto-triplet transition at the exchange energy $K$. The triplet-triplet coupling diverges at the Fermi energy if the triplets are degenerate, whereas the singlet is the nondegenerate ground state if $K>0$. The lifetime of the ground state is infinite if there is no current applied and thus the double quantum dot system is also a good case study for the effect of decoherence due to a finite current. If the current and therefore decoherence exceed a threshold as discussed in detail in Ref. 14, this leads to a non-equilibrium induced current through the exchange coupled quantum dot even in the linear response regime.

A further aspect of the model is the quantum phase transition inherent in a double quantum system due to the competition between the exchange coupling induced singlet ground state and the dynamic singlet state due to the Kondo interaction with the leads. For two impurities embedded in a metal a quantum phase transition occurs for $K \approx 2.2 T_{K}$, which is in a regime where the poor man's scaling approach breaks down. This quantum phase transition can be studied in more detail in the flow equation approach since i) the two-loop equation (94) contains the competition between singlet formation of the two dots vs. individual Kondo screening and ii) the Kondo coupling decreases below the decoherence scale in non-equilibrium. In order to do so a physical quantity like the spin susceptibility will be studied in a different publication 27 . Such a calculation is based on the key feature of the flow equation approach that decoherence and interaction effects, e.g. the spin-spin interaction in the double-dot system, are dealt with on the same footing.

We would like to thank J. Paaske, P. Wölfle and H. Schoeller for useful discussions and input at various stages of this project. Furthermore we would like to thank Ch. Bruder, B. Braunecker, K. Flensberg, S. Andergassen, D. Schuricht and L. Fritz for valuable discussions.

S. K. acknowledges support through SFB 631 of the Deutsche Forschungsgemeinschaft (DFG), the Center for Nanoscience (CeNS) Munich, and the German Excellence Initiative via the Nanosystems Initiative Munich (NIM).

\section{Appendix A: One-loop expressions}

With the expression for $\eta$ in Eq. (37) and inserting it into the flow equation (35), we find the scaling equation 


$$
\begin{aligned}
\frac{d J_{k^{\prime}}^{\mathrm{sum}, j}(B)}{d B}= & -\left(\epsilon_{k^{\prime}}-\epsilon_{k}\right)^{2} J_{k^{\prime} k}^{\mathrm{sum}, j} \\
& -\frac{1}{2} \sum_{q}((1-n(q j))-n(q j))\left(\left(\epsilon_{k^{\prime}}-\epsilon_{q}\right)-\left(\epsilon_{q}-\epsilon_{k}\right)\right) J_{k^{\prime} q}^{\mathrm{sum}, j} J_{q k}^{\mathrm{sum}, j} \\
& -\sum_{q}(1-n(q j))\left(\left(\epsilon_{k^{\prime}}-\epsilon_{q}+K\right)-\left(\epsilon_{q}-\epsilon_{k}-K\right)\right) 2 P_{k^{\prime} q}^{j} m_{q k}^{j} \\
& +\sum_{q} n(q j)\left(\left(\epsilon_{k^{\prime}}-\epsilon_{q}-K\right)-\left(\epsilon_{q}-\epsilon_{k}+K\right)\right) 2 m_{k^{\prime} q}^{j} P_{q k}^{j} \\
\frac{d P_{k^{\prime} k}^{j}(B)}{d B}= & -\left(\epsilon_{k^{\prime}}-\epsilon_{k}+K\right)^{2} P_{k^{\prime} k}^{j} \\
& -\sum_{q}(1-n(q j))\left(\left(\epsilon_{k^{\prime}}-\epsilon_{q}\right)-\left(\epsilon_{q}-\epsilon_{k}+K\right)\right) J_{k^{\prime} q}^{\mathrm{sum}, j} P_{q k}^{j} \\
& +\sum_{q} n(q j)\left(\left(\epsilon_{k^{\prime}}-\epsilon_{q}+K\right)-\left(\epsilon_{q}-\epsilon_{k}\right)\right) P_{k^{\prime} q}^{j} J_{q k}^{\mathrm{sum}, j}
\end{aligned}
$$

With the diagonal parametrization as defined in the main text we can write

$$
\begin{aligned}
& \frac{d J_{k}^{\mathrm{sum}, j}(B)}{d B}= \\
& -\sum_{q}(1-2 n(q j))\left(\epsilon_{k}-\epsilon_{q}\right) \mathrm{e}^{-2 B\left(\epsilon_{k}-\epsilon_{q}\right)^{2}}\left(J_{(k+q) / 2}^{\mathrm{sum}, j}\right)^{2} \\
& -4 \sum_{q}(1-n(q j))\left(\epsilon_{k}-\epsilon_{q}+K\right) \mathrm{e}^{-2 B\left(\epsilon_{k}-\epsilon_{q}+K\right)^{2}}\left(P_{(k+q) / 2}^{j}\right)^{2} \\
& +4 \sum_{q} n(q j)\left(\epsilon_{k}-\epsilon_{q}-K\right) \mathrm{e}^{-2 B\left(\epsilon_{k}-\epsilon_{q}-K\right)^{2}}\left(P_{(k+q) / 2}^{j}\right)^{2}
\end{aligned}
$$

$$
\begin{aligned}
& \frac{d P_{k}^{j}(B)}{d B}= \\
& -\sum_{q}(1-n(q j))\left(2\left(\epsilon_{k}-\epsilon_{q}-K / 2\right)\right) \mathrm{e}^{-2 B\left(\epsilon_{k}-\epsilon_{q}-K / 2\right)^{2}} \\
& \quad \times J_{(k-K / 2+q) / 2}^{\mathrm{sum}, j} P_{(q+k+K / 2) / 2}^{j} \\
& +\sum_{q} n(q j)\left(2\left(\epsilon_{k}-\epsilon_{q}+K / 2\right)\right) \mathrm{e}^{-B\left(\epsilon_{k}-\epsilon_{q}+K / 2\right)^{2}} \\
& \quad \times P_{(k-K / 2+q) / 2}^{j} J_{(q+k+K / 2) / 2}^{\mathrm{sum}, j}
\end{aligned}
$$

We assume the exponential dependence on the energy is stronger than the energy dependence of the coupling function. Doing the integration assuming that $\mathrm{e}^{-2 B(x-c)^{2}} f(x) \approx \mathrm{e}^{-2 B(x-c)^{2}} f(c)$ and neglecting contributions from $B_{0}=e^{-B \Lambda_{0}^{2}}$ we find 


$$
\begin{aligned}
\frac{d g_{k}^{\text {sum }, j}(B)}{d B} \approx & \frac{1}{2 B}\left(\frac{r_{j}}{1+r_{j}} \mathrm{e}^{-2 B\left(\epsilon_{k}-V_{j} / 2\right)^{2}}+\frac{1}{1+r_{j}} \mathrm{e}^{-2 B\left(\epsilon_{k}+V_{j} / 2\right)^{2}}\right)\left(g_{k}^{\text {sum }, j}\right)^{2} \\
& +2 \frac{1}{2 B}\left(\frac{r_{j}}{1+r_{j}} \mathrm{e}^{-2 B\left(\epsilon_{k}-V_{j} / 2+K\right)^{2}}+\frac{r_{j}}{1+r_{j}} \mathrm{e}^{-2 B\left(\epsilon_{k}-V_{j} / 2+K\right)^{2}}\right)\left(p_{k+K / 2}^{j}\right)^{2} \\
& +2 \frac{1}{2 B}\left(\frac{r_{j}}{1+r_{j}} \mathrm{e}^{-2 B\left(\epsilon_{k}-V_{j} / 2-K\right)^{2}}+\frac{1}{1+r_{j}} \mathrm{e}^{-2 B\left(\epsilon_{k}+V_{j} / 2-K\right)^{2}}\right)\left(p_{k-K / 2}^{j}\right)^{2} \\
\frac{d p_{k}^{j}(B)}{d B}= & \frac{1}{2 B}\left(\frac{r_{j}}{1+r_{j}} \mathrm{e}^{-2 B\left(\epsilon_{k}-V_{j} / 2-K / 2\right)^{2}}+\frac{1}{1+r_{j}} \mathrm{e}^{-2 B\left(\epsilon_{k}+V_{j} / 2-K / 2\right)^{2}}\right) g_{k-K / 2}^{\text {sum }, j} p_{k}^{j} \\
& +\frac{1}{2 B}\left(\frac{r_{j}}{1+r_{j}} \mathrm{e}^{-2 B\left(\epsilon_{k}-V_{j} / 2+K / 2\right)^{2}}+\frac{1}{1+r_{j}} \mathrm{e}^{-2 B\left(\epsilon_{k}+V_{j} / 2+K / 2\right)^{2}}\right) p_{k}^{j} g_{k+K / 2}^{\text {sum }, j}
\end{aligned}
$$

where we have now introduced the dimensionless couplings $g_{k^{\prime} k}^{\text {sum }, j}=N(0) J_{k^{\prime} k}^{\text {sum }, j}$ and $p_{k^{\prime} k}^{j}=N(0) P_{k^{\prime} k}^{j}$. This expression is for $r_{j}=1$ discussed in detail in the main text.

\section{Appendix B: Flow of the RKKY interaction}

In second order in the Kondo coupling we also generate an RKKY-like spin exchange interaction, $I_{R K K Y} \vec{S}_{L} \vec{S}_{R}$. Together with the flow of $I_{R K K Y}$

$$
\begin{aligned}
\frac{d}{d B} I_{R K K Y}=\sum_{k^{\prime} k}\{ & {\left[n\left(k^{\prime} j\right)-n(k j)\right] \eta_{k^{\prime} k}^{\mathrm{sum}, j} J_{k k^{\prime}}^{\mathrm{sum}, j} } \\
- & {\left[n\left(k^{\prime} j\right)-n(k j)\right] } \\
& \times\left(2 \eta_{k^{\prime} k}^{P j} M_{k k^{\prime}}^{j}+2 \eta_{k^{\prime} k}^{M j} P_{k k^{\prime}}^{j}\right) \\
+ & {\left[n(k j)\left(1-n\left(k^{\prime} j\right)\right)+n\left(k^{\prime} j\right)(1-n(k j))\right] } \\
& \left.\times\left(4 \eta_{k^{\prime} k}^{P j} M_{k k^{\prime}}^{j}-4 \eta_{k^{\prime} k}^{M j} P_{k k^{\prime}}^{j}\right)\right\} \quad(\mathrm{B} 1)
\end{aligned}
$$

there is also a constant proportional to $3 / 4 \hbar^{2}$ generated in the flow to second order.

$$
\begin{aligned}
\frac{d}{d B} E_{\text {const }}= & \frac{3}{4} \hbar^{2} \sum_{k^{\prime} k}\left\{\left[n\left(k^{\prime} j\right)-n(k j)\right] \eta_{k^{\prime} k}^{\mathrm{sum}, j} J_{k k^{\prime}}^{\mathrm{sum}, j}\right. \\
& \left.+\left[n\left(k^{\prime} j\right)-n(k j)\right]\left(2 \eta_{k^{\prime} k}^{P j} M_{k k^{\prime}}^{j}+2 \eta_{k^{\prime} k}^{M j} P_{k k^{\prime}}^{j}\right)\right\}
\end{aligned}
$$

In the following we study only the case for zero voltages, $V_{L}=V_{R}=0$.

If we now insert the diagonal parametrization for the couplings and do the energy integration assuming like before that the energy dependence of the couplings can be neglected. The integration over momentum yields

$$
\begin{aligned}
& \int d \epsilon_{k^{\prime}} \int d \epsilon_{k}\left[n(k j)\left(1-n\left(k^{\prime} j\right)\right)\right] \\
& \times\left(\epsilon_{k^{\prime}}-\epsilon_{k}+\alpha K\right) \mathrm{e}^{-2 B\left(\epsilon_{k^{\prime}}-\epsilon_{k}+\alpha K\right)^{2}} \\
& =\frac{\sqrt{2 \pi}}{16 B^{3 / 2}}(1-\operatorname{erf}(\sqrt{2 B} \alpha K)) \\
& \int d \epsilon_{k^{\prime}} \int d \epsilon_{k}\left[n\left(k^{\prime} j\right)(1-n(k j))\right] \\
& \quad \times\left(\epsilon_{k^{\prime}}-\epsilon_{k}+\alpha K\right) \mathrm{e}^{-2 B\left(\epsilon_{k^{\prime}}-\epsilon_{k}+\alpha K\right)^{2}} \\
& =-\frac{\sqrt{2 \pi}}{16 B^{3 / 2}}(1+\operatorname{erf}(\sqrt{2 B} \alpha K))
\end{aligned}
$$

Utilizing these results we thus find

$$
\begin{gathered}
\frac{d}{d B} I_{R K K Y}=\frac{\sqrt{2 \pi}}{8 B^{3 / 2}}\left\{[1-2 \operatorname{erf}(\sqrt{2 B} K)]\left(2 p_{\max }^{j}\right)^{2}\right. \\
\left.-\left(g_{\max }^{\mathrm{sum}, j}\right)^{2}\right\} \\
\frac{d}{d B} E_{\text {const }}=\frac{3}{4} \hbar^{2} \frac{\sqrt{2 \pi}}{8 B^{3 / 2}}\left\{-\left(g_{\text {max }}^{\text {sum } j}\right)^{2}-\left(2 p_{\text {max }}^{j}\right)^{2}\right\}
\end{gathered}
$$

where $g_{\max }^{\mathrm{sum}, j}$ and $p_{\max }^{j}$ are the couplings at the most divergent energy argument.

Note that for $B K^{2} \gg 1$ where $\operatorname{erf}(\sqrt{2 B} K) \approx 1$, the following relation holds

$$
\frac{3}{4} \hbar^{2} \frac{d I_{R K K Y}}{d B}-\frac{d E_{\text {const }}}{d B}=0
$$

i.e. the combination of the two newly generated couplings stops to flow as soon as $B$ has reached the energy scale $1 / K^{2}$. Corrections to each of the couplings $I_{R K K Y}$ and $E_{\text {const }}$ are given by a term proportional to $B^{-3 / 2}$ which is negligible small compared to the logarithmic divergence for small values of $B$. 
Furthermore in the opposite limit $B K^{2} \ll 1$

$$
\begin{aligned}
& \frac{d I_{R K K Y}}{d B} \\
& \approx-\frac{\sqrt{2 \pi}}{8 B^{3 / 2}}\left\{\left[-1+2 \frac{2}{\sqrt{\pi}} \sqrt{2 B} K\right]\left(2 p_{\max }^{j}\right)^{2}+\left(g_{\max }^{\text {sum }, j}\right)^{2}\right\} \\
& \approx-\frac{K}{B}\left(2 p_{\text {max }}^{j}\right)^{2}
\end{aligned}
$$

where we neglect the small term $\propto 1 / B^{3 / 2}$.

Note that the frequency dependence of the coupling functions is not developed in the limit $B K^{2} \gg 1$ and thus we find that the relation $g_{\max }^{\mathrm{sum}, j}=2 p_{\max }^{j}$ is fulfilled during the flow. Thus for example the coupling $g_{\max }^{\mathrm{sum}, j}$ fulfills the flow equation

$$
\begin{aligned}
\frac{d g_{\max }^{\mathrm{sum}, j}}{d B} & =\frac{1}{B}\left(g_{\max }^{\mathrm{sum}, j}\right)^{2} \\
\Rightarrow \quad g_{\max }^{\mathrm{sum}, j} & =\frac{1}{\ln \left(B T_{K}^{2}\right)}
\end{aligned}
$$

where $T_{K}$ is the Kondo temperature as defined in the main text. In the following we assume that the initial value $K$ on the right hand side of the flow equation is also subject of the flow and thus replace: $K \rightarrow I_{R K K Y}$. Consequently we have to solve the differential equation

$$
d \ln I_{R K K Y} \approx-2\left(\frac{1}{\ln \left(B T_{K}^{2}\right)}\right)^{2} d \ln B
$$

where the factor of 2 originates from the summation over the two lead indices $j$ which is special to the chosen model. The latter equation can be solved immediately and yields

$$
I_{R K K Y}(B)=K\left(1+\frac{1}{\ln \left(\sqrt{B} T_{K}\right)}-\frac{1}{\ln \left(\sqrt{B_{0}} T_{K}\right)}\right)
$$

where the initial value is given by $I_{R K K Y}\left(B_{0}\right)=K$ and $B_{0}=1 / \Lambda_{0}^{2}$. The above sketched calculation is only valid for $B K^{2} \ll 1$ but as we have also argued the flow for $B K^{2} \gg 1$ is negligible and thus we can write

$$
I_{R K K Y}(B \rightarrow \infty) \approx K\left(1-\left(\frac{1}{\ln \left(K / T_{K}\right)}-\frac{1}{\ln \left(\Lambda_{0} / T_{K}\right)}\right)\right)
$$

The calculation in this appendix illustrates that there are terms which lead to a logarithmic correction of the exchange energy gap. The renormalization of the quantum dot energy levels is known and occurs for example as the Knight shift for a spin-1/2 quantum dot in magnetic field ${ }^{7}$.

Note that there is no further contribution to $I_{R K K Y}$ from the two-loop calculation.

\section{Appendix C: two-loop}

The new interaction terms in $H_{\text {int }}^{(2)}$ as defined in Eq. (84) are generated due to two Kondo spin scattering processes

$$
\begin{aligned}
\frac{d\left(K_{k^{\prime} k ; q^{\prime} q}^{\mathrm{sum}}-K_{q^{\prime} q ; k^{\prime} k}^{\text {sum }}\right)}{d B} & =-\left(\epsilon_{k^{\prime}}-\epsilon_{k}+\epsilon_{q^{\prime}}-\epsilon_{q}\right)^{2}\left(K_{k^{\prime} k ; q^{\prime} q}^{\text {sum }}-K_{q^{\prime} q ; k^{\prime} k}^{\text {sum }}\right) \\
& +\left(\left(\epsilon_{k^{\prime}}-\epsilon_{k}\right)-\left(\epsilon_{q^{\prime}}-\epsilon_{q}\right)\right) J_{k^{\prime} k}^{\text {sum }, j} J_{q^{\prime} q}^{\text {sum }, v} \\
& +2\left(\left(\epsilon_{k^{\prime}}-\epsilon_{k}+K\right)-\left(\epsilon_{q^{\prime}}-\epsilon_{q}-K\right)\right) P_{k^{\prime} k}^{j} M_{q^{\prime} q}^{v} \\
& +2\left(\left(\epsilon_{k^{\prime}}-\epsilon_{k}-K\right)-\left(\epsilon_{q^{\prime}}-\epsilon_{q}+K\right)\right) M_{k^{\prime} k}^{j} P_{q^{\prime} q}^{v} \\
\frac{d\left(K_{k^{\prime} k ; q^{\prime} q}^{P}-K_{q^{\prime} q ; k^{\prime} k}^{P}\right)}{d B}= & -\left(\epsilon_{k^{\prime}}-\epsilon_{k}+\epsilon_{q^{\prime}}-\epsilon_{q}+K\right)^{2}\left(K_{k^{\prime} k ; q^{\prime} q}^{P}-K_{q^{\prime} q ; k^{\prime} k}^{P}\right) \\
+ & \left(\left(\epsilon_{k^{\prime}}-\epsilon_{k}\right)-\left(\epsilon_{q^{\prime}}-\epsilon_{q}+K\right)\right) J_{k^{\prime} k}^{\text {sum }, j} P_{q^{\prime} q}^{v} \\
+ & \left(\left(\epsilon_{k^{\prime}}-\epsilon_{k}+K\right)-\left(\epsilon_{q^{\prime}}-\epsilon_{q}\right)\right) P_{k^{\prime} k}^{j} J_{q^{\prime} q}^{\text {sum }, v} \\
\frac{d\left(K_{k^{\prime} k ; q^{\prime} q}^{M}-K_{q^{\prime} q ; k^{\prime} k}^{M}\right)}{d B}= & -\left(\epsilon_{k^{\prime}}-\epsilon_{k}+\epsilon_{q^{\prime}}-\epsilon_{q}-K\right)^{2}\left(K_{k^{\prime} k ; q^{\prime} q}^{M}-K_{q^{\prime} q ; k^{\prime} k}^{M}\right) \\
+ & \left(\left(\epsilon_{k^{\prime}}-\epsilon_{k}\right)-\left(\epsilon_{q^{\prime}}-\epsilon_{q}-K\right)\right) J_{k^{\prime} k}^{\text {sum }, j} M_{q^{\prime} q}^{v} \\
+ & \left(\left(\epsilon_{k^{\prime}}-\epsilon_{k}-K\right)-\left(\epsilon_{q^{\prime}}-\epsilon_{q}\right)\right) M_{k^{\prime} k}^{j} J_{q^{\prime} q}^{\mathrm{sum}, v}
\end{aligned}
$$




$$
\begin{aligned}
\frac{d\left(K_{k^{\prime} k ; q^{\prime} q}^{R K K}+K_{q^{\prime} q ; k^{\prime} k}^{R K K}\right)}{d B} & =-\left(\epsilon_{k^{\prime}}-\epsilon_{k}+\epsilon_{q^{\prime}}-\epsilon_{q}\right)^{2}\left(K_{k^{\prime} k ; q^{\prime} q}^{R K K}+K_{q^{\prime} q ; k^{\prime} k}^{R K Y}\right) \\
& -2\left(\left(\epsilon_{k^{\prime}}-\epsilon_{k}+K\right)-\left(\epsilon_{q^{\prime}}-\epsilon_{q}-K\right)\right) P_{k^{\prime} k}^{j} M_{q^{\prime} q}^{v} \\
& +2\left(\left(\epsilon_{k^{\prime}}-\epsilon_{k}-K\right)-\left(\epsilon_{q^{\prime}}-\epsilon_{q}+K\right)\right) M_{k^{\prime} k}^{j} P_{q^{\prime} q}^{v}
\end{aligned}
$$

Exchange of summation indices provides us with a symmetry constraint

$$
\begin{aligned}
K_{k^{\prime} k ; q^{\prime} q}^{\mathrm{sum}} & =-K_{q^{\prime} q ; k^{\prime} k}^{\mathrm{sum}}, \\
K_{k^{\prime} k ; q^{\prime} q}^{P / M} & =-K_{q^{\prime} q ; k^{\prime} k}^{P / M}, \\
K_{k^{\prime} k ; q^{\prime} q}^{R K K} & =K_{q^{\prime} q ; k^{\prime} k}^{R K K} .
\end{aligned}
$$

In addition the hermiticity of the Hamiltonian has to be fulfilled and such there are some simplifying relations

$$
\begin{aligned}
K_{k^{\prime} k ; q^{\prime} q}^{\text {sum }} & =-K_{k k^{\prime} ; q q^{\prime}}^{\text {sum }} \\
K_{k^{\prime} k ; q^{\prime} q}^{P / M} & =-K_{k k^{\prime} ; q q^{\prime}}^{M / P} \\
K_{k^{\prime} k ; q^{\prime} q}^{R K Y} & =K_{k k^{\prime} ; q q^{\prime}}^{R K Y}
\end{aligned}
$$

These relations are also fulfilled in the scaling equations.

The canonical generator $\eta^{(2)}=\left[H_{0}, H_{i n t}^{2}\right]$ in second order of the Kondo coupling is explicitly given by

$$
\begin{aligned}
\eta_{i n t}^{(2)}= & \left(\epsilon_{k^{\prime}}-\epsilon_{k}+\epsilon_{q^{\prime}}-\epsilon_{q}\right) K_{k^{\prime} k ; q^{\prime} q}^{\operatorname{sum}_{i}} i:\left(\vec{S}_{L}+\vec{S}_{R}\right)\left(\vec{s}_{\left(k^{\prime} j\right)(k j)} \times \vec{s}_{\left(q^{\prime} v\right)(q v)}\right): \\
& +\left(\epsilon_{k^{\prime}}-\epsilon_{k}+\epsilon_{q^{\prime}}-\epsilon_{q}+K\right) K_{k^{\prime} k ; q^{\prime} q}^{P} i:\left(\left(\vec{S}_{L}-\vec{S}_{R}\right)+2 i\left(\vec{S}_{L} \times \vec{S}_{R}\right)\right)\left(\vec{s}_{\left(k^{\prime} j\right)(k j)} \times \vec{s}_{\left(q^{\prime} v\right)(q v)}\right): \\
& +\left(\epsilon_{k^{\prime}}-\epsilon_{k}+\epsilon_{q^{\prime}}-\epsilon_{q}-K\right) K_{k^{\prime} k ; q^{\prime} q}^{M} i:\left(\left(\vec{S}_{L}-\vec{S}_{R}\right)-2 i\left(\vec{S}_{L} \times \vec{S}_{R}\right)\right)\left(\vec{s}_{\left(k^{\prime} j\right)(k j)} \times \vec{s}_{\left(q^{\prime} v\right)(q v)}\right): \\
& +\left(\epsilon_{k^{\prime}}-\epsilon_{k}+\epsilon_{q^{\prime}}-\epsilon_{q}\right) K_{k^{\prime} k ; q^{\prime} q}^{R K Y} i 2 i:\left(\vec{S}_{L} \times \vec{s}_{\left(k^{\prime} j\right)(k j)}\right)\left(\vec{S}_{R} \times \vec{s}_{\left(q^{\prime} v\right)(q v)}\right)+\left(\vec{S}_{R} \times \vec{s}_{\left(k^{\prime} j\right)(k j)}\right)\left(\vec{S}_{L} \times \vec{s}_{\left(q^{\prime} v\right)(q v)}\right): \\
& =\eta_{(2)}^{\text {sum }}+\eta_{(2)}^{P}+\eta_{(2)}^{M}+\eta_{(2)}^{R K K Y}
\end{aligned}
$$

Note that both $K^{\mathrm{sum}}$ and $K^{R K K Y}$ do not involve a singlet-triplet transition like $K^{P / M}$.

From $\left[\eta^{(2)}, H^{(1)}\right]$ and $\left[\eta^{(1)}, H^{(2)}\right]$ we find the higher order contributions to the flow $d H / d B$ and thus a scaling equation for the coupling $g_{k^{\prime} k}^{\text {sum,j }}$ to two-loop order

$$
\begin{aligned}
\frac{d g_{k^{\prime} k}^{\mathrm{sum}, j}}{d B} & =\ldots \\
& +\frac{1}{2} \sum_{q^{\prime} q}\left[n\left(q^{\prime} v\right)(1-n(q v))+n(q v)\left(1-n\left(q^{\prime} v\right)\right)\right]\left(\epsilon_{k^{\prime}}-\epsilon_{k}+\epsilon_{q^{\prime}}-\epsilon_{q}-\left(\epsilon_{q}-\epsilon_{q^{\prime}}\right)\right)\left(k_{k^{\prime} k ; q^{\prime} q}^{\mathrm{sum}}-k_{q^{\prime} q ; k^{\prime} k}^{\mathrm{sum}}\right) g_{q q^{\prime}}^{\mathrm{sum}, v} \\
& +\frac{1}{2} \sum_{q^{\prime} q}\left(n\left(q^{\prime} v\right)-n(q v)\right)\left(\epsilon_{k^{\prime}}-\epsilon_{k}+\epsilon_{q^{\prime}}-\epsilon_{q}-\left(\epsilon_{q}-\epsilon_{q^{\prime}}\right)\right)\left(k_{k^{\prime} k ; q^{\prime} q}^{R K K}+k_{q^{\prime} q ; k^{\prime} k}^{R K K}\right) g_{q q^{\prime}}^{\mathrm{sum}, v} \\
& +2 \sum_{q^{\prime} q} n(q v)\left(1-n\left(q^{\prime} v\right)\right)\left(\epsilon_{k^{\prime}}-\epsilon_{k}+\epsilon_{q^{\prime}}-\epsilon_{q}-K-\left(\epsilon_{q}-\epsilon_{q^{\prime}}+K\right)\right)\left(k_{k^{\prime} k ; q^{\prime} q}^{M}-k_{q^{\prime} q ; k^{\prime} k}^{M}\right) p_{q q^{\prime}}^{v} \\
& +2 \sum_{q^{\prime} q} n\left(q^{\prime} v\right)(1-n(q v))\left(\epsilon_{k^{\prime}}-\epsilon_{k}+\epsilon_{q^{\prime}}-\epsilon_{q}+K-\left(\epsilon_{q}-\epsilon_{q^{\prime}}-K\right)\right)\left(k_{k^{\prime} k ; q^{\prime} q}^{P}-k_{q^{\prime} q ; k^{\prime} k}^{P}\right) m_{q q^{\prime}}^{v}
\end{aligned}
$$

where $k_{k^{\prime} k ; q^{\prime} q}=N(0) K_{k^{\prime} k ; q^{\prime} q}$ and we neglected a contribution from

$$
-2 \sum_{q^{\prime} q}\left(n\left(q^{\prime} v\right)-n(q v)\right)\left(\epsilon_{k^{\prime}}-\epsilon_{k}+\epsilon_{q^{\prime}}-\epsilon_{q}-\left(\epsilon_{q}-\epsilon_{q^{\prime}}\right)\right)\left(k_{k^{\prime} q ; q^{\prime} k}^{R K K Y}+k_{q^{\prime} k ; k^{\prime} q}^{R K K Y}\right) g_{q q^{\prime}}^{\mathrm{sum}}:\left(\vec{S}_{L}+\vec{S}_{R}\right) \vec{s}_{\left(k^{\prime} j\right)(k j)}:
$$

It can be shown in the simplest limit that this term is proportional to $B^{-3 / 2}$ and thus negligible compared to the leading order $B^{-1 / 2}$. In the more general cases it can be shown numerically that the contribution from $k_{k^{\prime}} q ; q^{\prime} k$ does not have an effect on the flow. 
Furthermore the expression for $p_{k^{\prime} k}^{j}$ yields

$$
\begin{aligned}
\frac{d p_{k^{\prime} k}^{j}}{d B} & =\ldots \\
& +\sum_{q^{\prime} q} n(q v)\left(1-n\left(q^{\prime} v\right)\right)\left(\epsilon_{q^{\prime}}-\epsilon_{q}+\epsilon_{k^{\prime}}-\epsilon_{k}+K-\left(\epsilon_{q}-\epsilon_{q^{\prime}}\right)\right)\left(k_{k^{\prime} k ; q^{\prime} q}^{P}-k_{q^{\prime} q ; k^{\prime} k}^{P}\right) g_{q q^{\prime}}^{\text {sum }, v} \\
& +\sum_{q^{\prime} q} n\left(q^{\prime} v\right)(1-n(q v))\left(\epsilon_{q^{\prime}}-\epsilon_{q}+\epsilon_{k^{\prime}}-\epsilon_{k}-\left(\epsilon_{q}-\epsilon_{q^{\prime}}+K\right)\right)\left(k_{k^{\prime} k ; q^{\prime} q}^{\mathrm{sum}}-k_{q^{\prime} q ; k^{\prime} k}^{\mathrm{sum}}\right) p_{q q^{\prime}}^{v} \\
& -\sum_{q^{\prime} q} n\left(q^{\prime} v\right)(1-n(q v))\left(\epsilon_{q^{\prime}}-\epsilon_{q}+\epsilon_{k^{\prime}}-\epsilon_{k}-\left(\epsilon_{q}-\epsilon_{q^{\prime}}+K\right)\right)\left(k_{k^{\prime} k ; q^{\prime} q}^{R K K}+k_{q^{\prime} q ; k^{\prime} k}^{R K K}\right) p_{q q^{\prime}}^{v}
\end{aligned}
$$

where we neglect contributions from

$$
\begin{aligned}
& -\sum_{q^{\prime} q}\left[n\left(q^{\prime} v\right)(1-n(q v))+n(q v)\left(1-n\left(q^{\prime} v\right)\right)\right]\left(\left(\eta_{k^{\prime} k ; q^{\prime} q}^{R K K Y}+\eta_{q^{\prime} q ; k^{\prime} k}^{R K K Y}\right) P_{q q^{\prime}}^{v}-\left(K_{k^{\prime} k ; q^{\prime} q}^{R K K Y}+K_{q^{\prime} q ; k^{\prime} k}^{R K K}\right) \eta_{q q^{\prime}}^{P}\right) \\
& \times:\left(\left(\vec{S}_{L}-\vec{S}_{R}\right)+2 i\left(\vec{S}_{L} \times \vec{S}_{R}\right)\right) \vec{s}_{\left(k^{\prime} j\right)(k j)}: \\
& +2 \sum_{q^{\prime} q} n\left(q^{\prime} v\right)(1-n(q v))\left(\left(\eta_{k^{\prime} q ; q^{\prime} k}^{R K K}+\eta_{q^{\prime} k ; k^{\prime} q}^{R K Y}\right) P_{q q^{\prime}}^{v}-\left(K_{k^{\prime} q ; q^{\prime} k}^{R K K}+K_{q^{\prime} k ; k^{\prime} q}^{R K K}\right) \eta_{q q^{\prime}}^{P}\right) \\
& \quad \times:\left(\left(\vec{S}_{L}-\vec{S}_{R}\right)+2 i\left(\vec{S}_{L} \times \vec{S}_{R}\right)\right) \vec{s}_{\left(k^{\prime} j\right)(k j)}:
\end{aligned}
$$

This is a well-controlled approximation using the argument before that the terms proportional to $k^{\prime} q ; q^{\prime} k$ can be shown numerically to be negligible small compared to the terms of type $k^{\prime} k ; q^{\prime} q$. Additionally we can use the symmetry of the system that $p_{k^{\prime} k}^{L}=-p_{k^{\prime} k}^{R}$ and thus the first term in the latter equation cancels and can therefore be neglected.

Since we are not interested in the flow of the newly generated couplings, we integrate out the scaling equations for them in order to find their B-dependence. We illustrate this procedure on $K_{k^{\prime} k q^{\prime} q}^{\mathrm{sum}}$ as an example:

$$
\begin{aligned}
\left(K_{k^{\prime} k ; q^{\prime} q}^{\text {sum }}-K_{q^{\prime} q ; k^{\prime} k}^{\text {sum }}\right)(B)= & \mathrm{e}^{-B\left(\epsilon_{k^{\prime}}-\epsilon_{k}+\epsilon_{q^{\prime}}-\epsilon_{q}\right)^{2}} \int_{B_{0}}^{B} d B^{\prime} \mathrm{e}^{+B^{\prime}\left(\epsilon_{k^{\prime}}-\epsilon_{k}+\epsilon_{q^{\prime}}-\epsilon_{q}\right)^{2}} \\
& \left\{\left(\left(\epsilon_{k^{\prime}}-\epsilon_{k}\right)-\left(\epsilon_{q^{\prime}}-\epsilon_{q}\right)\right) J_{k^{\prime} k}^{\mathrm{sum}, j}\left(B^{\prime}\right) J_{q^{\prime} q}^{\mathrm{sum}, v}\left(B^{\prime}\right)\right. \\
& +2\left(\left(\epsilon_{k^{\prime}}-\epsilon_{k}+K\right)-\left(\epsilon_{q^{\prime}}-\epsilon_{q}-K\right)\right) P_{k^{\prime} k}^{j}\left(B^{\prime}\right) M_{q^{\prime} q}^{v}\left(B^{\prime}\right) \\
& \left.+2\left(\left(\epsilon_{k^{\prime}}-\epsilon_{k}-K\right)-\left(\epsilon_{q^{\prime}}-\epsilon_{q}+K\right)\right) M_{k^{\prime} k}^{j}\left(B^{\prime}\right) P_{q^{\prime} q}^{v}\left(B^{\prime}\right)\right\}
\end{aligned}
$$

In the following we apply the diagonal parametrization for the Kondo couplings and thus find

$$
\begin{aligned}
\left(K_{k^{\prime} k ; q^{\prime} q}^{\mathrm{sum}}-K_{q^{\prime} q ; k^{\prime} k}^{\text {sum }}\right)(B)=\mathrm{e}^{-B\left(\epsilon_{k^{\prime}}-\epsilon_{k}+\epsilon_{q^{\prime}}-\epsilon_{q}\right)^{2}} \int_{B_{0}}^{B} d B^{\prime} \mathrm{e}^{+B^{\prime}\left(\epsilon_{k^{\prime}}-\epsilon_{k}+\epsilon_{q^{\prime}}-\epsilon_{q}\right)^{2}} & \\
& \left\{\left(\left(\epsilon_{k^{\prime}}-\epsilon_{k}\right)-\left(\epsilon_{q^{\prime}}-\epsilon_{q}\right)\right) \mathrm{e}^{-B^{\prime}\left(\epsilon_{k^{\prime}}-\epsilon_{k}\right)^{2}} J_{k}^{\mathrm{sum}, j}\left(B^{\prime}\right) \mathrm{e}^{-B^{\prime}\left(\epsilon_{q^{\prime}}-\epsilon_{q}\right)^{2}} J_{q}^{\mathrm{sum}, v}\left(B^{\prime}\right)\right. \\
& +2\left(\left(\epsilon_{k^{\prime}}-\epsilon_{k}+K\right)-\left(\epsilon_{q^{\prime}}-\epsilon_{q}-K\right)\right) \mathrm{e}^{-B^{\prime}\left(\epsilon_{k^{\prime}}-\epsilon_{k}+K\right)^{2}} P_{k}^{j}\left(B^{\prime}\right) \mathrm{e}^{-B^{\prime}\left(\epsilon_{q^{\prime}}-\epsilon_{q}-K\right)^{2}} P_{q}^{v}\left(B^{\prime}\right) \\
& \left.+2\left(\left(\epsilon_{k^{\prime}}-\epsilon_{k}-K\right)-\left(\epsilon_{q^{\prime}}-\epsilon_{q}+K\right)\right) \mathrm{e}^{-B^{\prime}\left(\epsilon_{k^{\prime}}-\epsilon_{k}-K\right)^{2}} P_{k}^{j}\left(B^{\prime}\right) \mathrm{e}^{-B^{\prime}\left(\epsilon_{q^{\prime}}-\epsilon_{q}+K\right)^{2}} P_{q}^{v}\left(B^{\prime}\right)\right\}
\end{aligned}
$$

In the calculation to one-loop order we found that the Kondo couplings depend logarithmically on the flow parameter and thus have a much slower dependence than the exponential function in the latter expression. In the following we therefore assume that we can replace the coupling by their averaged value in the interval from $B_{0}$ to $B$. In general the initial value $B_{0}=1 / \Lambda_{0}^{2}$ should be chosen as the band cutoff in order to treat boundary terms. Since this is equivalent to treating a different model with new boundary conditions we set in the following as also in the main text

$$
B_{0}=0
$$


Using these two simplifications the integration is simple and can be done straightforwardly yielding

$$
\begin{aligned}
\left(K_{k^{\prime} k ; q^{\prime} q}^{\text {sum }}-K_{q^{\prime} q ; k^{\prime} k}^{\text {sum }}\right)(B)=\frac{1}{2 B} & \mathrm{e}^{-B\left(\epsilon_{k^{\prime}}-\epsilon_{k}+\epsilon_{q^{\prime}}-\epsilon_{q}\right)^{2}} \\
& \left\{\frac{\left(\epsilon_{k^{\prime}}-\epsilon_{k}\right)-\left(\epsilon_{q^{\prime}}-\epsilon_{q}\right)}{\left(\epsilon_{k^{\prime}}-\epsilon_{k}\right)\left(\epsilon_{q^{\prime}}-\epsilon_{q}\right)}\left(\mathrm{e}^{2 B\left(\epsilon_{k^{\prime}}-\epsilon_{k}\right)\left(\epsilon_{q^{\prime}}-\epsilon_{q}\right)}-1\right)\left[\frac{1}{B} \int_{0}^{B} d B^{\prime} J_{k}^{\mathrm{sum}, j}\left(B^{\prime}\right) J_{q}^{\mathrm{sum}, v}\left(B^{\prime}\right)\right]\right. \\
& +2 \frac{\left(\epsilon_{k^{\prime}}-\epsilon_{k}+K\right)-\left(\epsilon_{q^{\prime}}-\epsilon_{q}-K\right)}{\left(\epsilon_{k^{\prime}}-\epsilon_{k}+K\right)\left(\epsilon_{q^{\prime}}-\epsilon_{q}-K\right)}\left(\mathrm{e}^{2 B\left(\epsilon_{k^{\prime}}-\epsilon_{k}+K\right)\left(\epsilon_{q^{\prime}}-\epsilon_{q}-K\right)}-1\right)\left[\frac{1}{B} \int_{0}^{B} d B^{\prime} P_{k}^{j}\left(B^{\prime}\right) P_{q}^{v}\left(B^{\prime}\right)\right] \\
& \left.+2 \frac{\left(\epsilon_{k^{\prime}}-\epsilon_{k}-K\right)-\left(\epsilon_{q^{\prime}}-\epsilon_{q}+K\right)}{\left(\epsilon_{k^{\prime}}-\epsilon_{k}-K\right)\left(\epsilon_{q^{\prime}}-\epsilon_{q}+K\right)}\left(\mathrm{e}^{2 B\left(\epsilon_{k^{\prime}}-\epsilon_{k}-K\right)\left(\epsilon_{q^{\prime}}-\epsilon_{q}+K\right)}-1\right)\left[\frac{1}{B} \int_{0}^{B} d B^{\prime} P_{k}^{j}\left(B^{\prime}\right) P_{q}^{v}\left(B^{\prime}\right)\right]\right\}
\end{aligned}
$$

The same procedure is applied to the other coupling generated to two-loop order and these expressions are then inserted in the flow equation for the Kondo coupling. In the following we write down only the result for the diagonal parametrization where we assume e.g. in $g_{k}^{\text {sum,j }}$ that $\epsilon_{k^{\prime}}=\epsilon_{k}$. We find that the collected terms contributing to $d g_{k}^{\mathrm{sum}, j} / d B$ in two-loop order can be written by $T L\left(q, q^{\prime}\right)+T L\left(q^{\prime}, q\right)$ where

$$
\begin{aligned}
T L\left(q, q^{\prime}\right)= & \frac{1}{2} B \sum_{q^{\prime} q} n\left(q^{\prime} v\right)(1-n(q v))\left(-2\left(\epsilon_{q^{\prime}}-\epsilon_{q}\right)^{2}\right) \mathrm{e}^{-2 B\left(\epsilon_{q^{\prime}}-\epsilon_{q}\right)^{2}} \\
& {\left[\frac{1}{B} \int_{B_{0}}^{B} d B^{\prime} g_{k}^{\mathrm{sum}, j}\left(B^{\prime}\right) g_{\left(q^{\prime}+q\right) / 2}^{\mathrm{sum}, v}\left(B^{\prime}\right)\right] g_{\left(q+q^{\prime}\right) / 2}^{\mathrm{sum}, v}(B) } \\
+ & 2 B \sum_{q^{\prime} q} n\left(q^{\prime} v\right)(1-n(q v))\left(-2\left(\epsilon_{q^{\prime}}-\epsilon_{q}+K\right)^{2}\right) \mathrm{e}^{-2 B\left(\epsilon_{q^{\prime}}-\epsilon_{q}+K\right)^{2}} \\
& {\left[\frac{1}{B} \int_{B_{0}}^{B} d B^{\prime} g_{k}^{\mathrm{sum}, j}\left(B^{\prime}\right) p_{\left(q^{\prime}+q\right) / 2}^{v}\left(B^{\prime}\right)\right] p_{\left(q+q^{\prime}\right) / 2}^{v}(B) } \\
+ & 2 \sum_{q^{\prime} q}\left[n\left(q^{\prime} v\right)(1-n(q v))\right] \mathrm{e}^{-2 B\left(\epsilon_{q^{\prime}}-\epsilon_{q}\right)^{2}} \frac{\left(\epsilon_{q^{\prime}}-\epsilon_{q}+K\right)^{2}-K^{2}}{K\left(\epsilon_{q^{\prime}}-\epsilon_{q}+K\right)}\left(\mathrm{e}^{2 B(-K)\left(\epsilon_{q^{\prime}}-\epsilon_{q}+K\right)}-1\right) \\
& {\left[\frac{1}{B} \int_{B_{0}}^{B} d B^{\prime} p_{k}^{j}\left(B^{\prime}\right) p_{\left(q^{\prime}+q\right) / 2}^{v}\left(B^{\prime}\right)\right] g_{\left(q+q^{\prime}\right) / 2}^{\mathrm{sum}, v}(B) } \\
+ & 2 \sum_{q^{\prime} q}\left[n\left(q^{\prime} v\right)(1-n(q v))\right] \mathrm{e}^{-2 B\left(\epsilon_{q^{\prime}}-\epsilon_{q}+K\right)^{2}} \frac{K^{2}-\left(\epsilon_{q^{\prime}}-\epsilon_{q}\right)^{2}}{K\left(\epsilon_{q^{\prime}}-\epsilon_{q}\right)}\left(\mathrm{e}^{2 B(+K)\left(\epsilon_{q^{\prime}}-\epsilon_{q}\right)}-1\right) \\
& {\left[\frac{1}{B} \int_{B_{0}}^{B} d B^{\prime} p_{k}^{j}\left(B^{\prime}\right) g_{\left(q^{\prime}+q\right) / 2}^{\mathrm{sum}, v}\left(B^{\prime}\right)\right] p_{\left(q+q^{\prime}\right) / 2}^{v}(B) }
\end{aligned}
$$

Note that $T L\left(q, q^{\prime}\right)=T L\left(q^{\prime}, q\right)$. For the symmetric setup we are studying, only the first two terms are non-zero after summation over the lead index $\left(p_{k}^{L}=-p_{k}^{R}\right)$ and the last two terms are thus neglected in the following. Otherwise the last two terms are nasty whereas the first two terms can be summed over momentum straightforwardly.

Please note that the last two terms are of the same order than the first two terms if $K=0$. In the expressions which are discussed in the main text it is thus not valid to set $K=0$, especially in the case when one wants to derive the spin- $1 / 2$ limiting case where the cancellation of $p_{k}^{L}=-p_{k}^{R}$ does not take place. 
In $d p / d B$ the terms are less symmetric

$$
\begin{aligned}
\frac{d p_{k}^{j}(B)}{d B}= & \text { one-loop } \\
+ & B \sum_{q^{\prime} q} n(q v)\left(1-n\left(q^{\prime} v\right)\right)\left(-2\left(\epsilon_{q^{\prime}}-\epsilon_{q}\right)^{2}\right) \mathrm{e}^{-2 B\left(\epsilon_{q^{\prime}}-\epsilon_{q}\right)^{2}} \\
& {\left[\frac{1}{B} \int_{B_{0}}^{B} d B^{\prime} p_{k}^{j}\left(B^{\prime}\right) g_{\left(q^{\prime}+q\right) / 2}^{\text {sum },}\left(B^{\prime}\right)\right] g_{\left(q+q^{\prime}\right) / 2}^{\text {sum }, v} } \\
+ & 4 B \sum_{q^{\prime} q} n\left(q^{\prime} v\right)(1-n(q v))\left(-2\left(\epsilon_{q^{\prime}}-\epsilon_{q}-K\right)^{2}\right) \mathrm{e}^{-2 B\left(\epsilon_{q^{\prime}}-\epsilon_{q}-K\right)^{2}} \\
& {\left[\frac{1}{B} \int_{B_{0}}^{B} d B^{\prime} p_{k}^{j}\left(B^{\prime}\right) p_{\left(q^{\prime}+q\right) / 2}^{v}\left(B^{\prime}\right)\right] p_{\left(q+q^{\prime}\right) / 2}^{v} } \\
+ & \sum_{q^{\prime} q} n\left(q^{\prime} v\right)(1-n(q v)) \mathrm{e}^{-2 B\left(\epsilon_{q^{\prime}}-\epsilon_{q}-K\right)^{2}} \frac{\left(\epsilon_{q^{\prime}}-\epsilon_{q}\right)^{2}-K^{2}}{K\left(\epsilon_{q^{\prime}}-\epsilon_{q}\right)}\left(\mathrm{e}^{2 B(-K)\left(\epsilon_{q^{\prime}}-\epsilon_{q}\right)}-1\right) \\
& {\left[\frac{1}{B} \int_{B_{0}}^{B} d B^{\prime} g_{k}^{\text {sum }, j}\left(B^{\prime}\right) g_{\left(q^{\prime}+q\right) / 2}^{\text {sum }, v}\left(B^{\prime}\right)\right] p_{\left(q+q^{\prime}\right) / 2}^{v} } \\
+ & \sum_{q^{\prime} q} n(q v)\left(1-n\left(q^{\prime} v\right)\right) \mathrm{e}^{-2 B\left(\epsilon_{q^{\prime}}-\epsilon_{q}\right)^{2}} \frac{\left(\epsilon_{q^{\prime}}-\epsilon_{q}+K\right)^{2}-K^{2}}{K\left(\epsilon_{q^{\prime}}-\epsilon_{q}+K\right)}\left(\mathrm{e}^{2 B(-K)\left(\epsilon_{q^{\prime}}-\epsilon_{q}+K\right)}-1\right) \\
& {\left[\frac{1}{B} \int_{B_{0}}^{B} d B^{\prime} g_{k}^{\text {sum }, j}\left(B^{\prime}\right) p_{\left(q^{\prime}+q\right) / 2}^{v}\left(B^{\prime}\right)\right] g_{\left(q+q^{\prime}\right) / 2}^{\text {sum }, v} }
\end{aligned}
$$

Note that we do not have $q \leftrightarrow q^{\prime}$ symmetry! This is somehow expected since $p_{k^{\prime} k}$ is only symmetric under exchange of $k \leftrightarrow k^{\prime}$ and also $K \leftrightarrow-K$. The latter symmetry is fulfilled in the scaling equation.

For the energy integration the following relation is useful

$$
\begin{aligned}
\int d \epsilon_{q^{\prime}} \int & d \epsilon_{q} n(q v)\left(1-n\left(q^{\prime} v\right)\right) \\
& \times\left(\epsilon_{q^{\prime}}-\epsilon_{q}+\alpha K\right)^{2} \mathrm{e}^{-2 B\left(\epsilon_{q^{\prime}}-\epsilon_{q}+\alpha K\right)^{2}} \\
=\left(1-\frac{2 r_{v}}{\left(1+r_{v}\right)^{2}}\right) F(\alpha K) & \\
+ & \frac{r_{v}}{\left(1+r_{v}\right)^{2}}\left(F\left(\alpha K+e V_{v}\right)+F\left(\alpha K-e V_{v}\right)\right)
\end{aligned}
$$

where contributions from the band cutoff cancel out or are exponentially small and

$$
F(y)=\frac{y}{8 B} \frac{\sqrt{\pi}}{\sqrt{2 B}} \operatorname{erf}(\sqrt{2 B} y)+\frac{1}{2(2 B)^{2}} \mathrm{e}^{-2 B y^{2}} .
$$

The explicit expression for $d g_{k}^{\text {sum, } j} / d B$ after integration is given in the main text. 


$$
\begin{aligned}
\frac{d g_{k}^{\text {sum }, j}(B)}{d B}= & \frac{1}{2 B}\left(\frac{r_{j}}{1+r_{j}} \mathrm{e}^{-2 B\left(\epsilon_{k}-V_{j} / 2\right)^{2}}+\frac{1}{1+r_{j}} \mathrm{e}^{-2 B\left(\epsilon_{k}+V_{j} / 2\right)^{2}}\right)\left(g_{k}^{\text {sum }, j}\right)^{2} \\
& +2 \frac{1}{2 B}\left(\frac{r_{j}}{1+r_{j}} \mathrm{e}^{-2 B\left(\epsilon_{k}-V_{j} / 2+K\right)^{2}}+\frac{r_{j}}{1+r_{j}} \mathrm{e}^{-2 B\left(\epsilon_{k}-V_{j} / 2+K\right)^{2}}\right)\left(p_{k+K / 2}^{j}\right)^{2} \\
& +2 \frac{1}{2 B}\left(\frac{r_{j}}{1+r_{j}} \mathrm{e}^{-2 B\left(\epsilon_{k}-V_{j} / 2-K\right)^{2}}+\frac{1}{1+r_{j}} \mathrm{e}^{-2 B\left(\epsilon_{k}+V_{j} / 2-K\right)^{2}}\right)\left(p_{k-K / 2}^{j}\right)^{2} \\
& -\sum_{v} \frac{1}{4 B} g_{k}^{\text {sum }, j}(B)\left(g_{\text {max }}^{\text {sum }, v}(B)\right)^{2} \\
& -\sum_{v} g\left(0, r_{v}, V_{v}\right) g_{k}^{\text {sum }, j}(B)\left(g_{\text {max }}^{\text {sum }, v}(B)\right)^{2} \\
& -\sum_{v}\left(\frac{1}{4 B} \mathrm{e}^{-2 B K^{2}}+\frac{K}{4} \sqrt{\frac{\pi}{2 B}} \operatorname{erf}(\sqrt{2 B} K)\right) g_{k}^{\text {sum }, j}(B)\left(2 p_{\text {max }}^{v}(B)\right)^{2} \\
& -\sum_{v} g\left(K, r_{v}, V_{v}\right) g_{k}^{\text {sum }, j}(B)\left(2 p_{\text {max }}^{v}(B)\right)^{2}
\end{aligned}
$$

and

$$
\begin{aligned}
\frac{d p_{k}^{j}(B)}{d B}= & \frac{1}{2 B}\left(\frac{r_{j}}{1+r_{j}} \mathrm{e}^{-2 B\left(\epsilon_{k}-V_{j} / 2-K / 2\right)^{2}}+\frac{1}{1+r_{j}} \mathrm{e}^{-2 B\left(\epsilon_{k}+V_{j} / 2-K / 2\right)^{2}}\right) g_{k-K / 2}^{\mathrm{sum}, j} p_{k}^{j} \\
& +\frac{1}{2 B}\left(\frac{r_{j}}{1+r_{j}} \mathrm{e}^{-2 B\left(\epsilon_{k}-V_{j} / 2+K / 2\right)^{2}}+\frac{1}{1+r_{j}} \mathrm{e}^{-2 B\left(\epsilon_{k}+V_{j} / 2+K / 2\right)^{2}}\right) p_{k}^{j} g_{k+K / 2}^{\mathrm{sum}, j} \\
& -\sum_{v} \frac{1}{4 B} p_{k}^{j}(B)\left(g_{\max }^{\mathrm{sum}, v}(B)\right)^{2} \\
& -\sum_{v} g\left(0, r_{v}, V_{v}\right) p_{k}^{j}(B)\left(g_{\max }^{\mathrm{sum}, v}(B)\right)^{2} \\
& -\sum_{v}\left(\frac{1}{4 B} \mathrm{e}^{-2 B K^{2}}+\frac{K}{4} \sqrt{\frac{\pi}{2 B}} \operatorname{erf}(\sqrt{2 B} K)\right) p_{k}^{j}(B)\left(2 p_{\max }^{v}(B)\right)^{2} \\
& -\sum_{v} g\left(K, r_{v}, V_{v}\right) p_{k}^{j}(B)\left(2 p_{\max }^{v}(B)\right)^{2}
\end{aligned}
$$

where

$$
\begin{aligned}
g(K, r, V) & =\frac{1}{4 B} \frac{r}{(1+r)^{2}}\left(\mathrm{e}^{-2 B(K-V)^{2}}+\mathrm{e}^{-2 B(K+V)^{2}}-2 \mathrm{e}^{-2 B K^{2}}\right) \\
& +\frac{1}{4} \sqrt{\frac{\pi}{2 B}} \frac{r}{(1+r)^{2}}((K-V) \operatorname{erf}(\sqrt{2 B}(K-V))+(K+V) \operatorname{erf}(\sqrt{2 B}(K+V))-2 K \operatorname{erf}(\sqrt{2 B} K))
\end{aligned}
$$

For $r=1$ the function $g(K, V)$ corresponds to the function $f(K, V)$ in the main text.

* author to whom correspondence should be addressed: koerting@nbi.dk

1 D. Goldhaber-Gordon, H. Shtrikman, D. Mahalu,D. Abusch-Magder, U. Meirav, and M. A. Kastner, Nature 391, 156 (1998); S. M. Cronenwett, T. H. Oosterkamp, and L. P. Kouwenhoven, Science 281, 540 (1998); J. Schmid, J. Weis, K. Eberl, and K. v. Klitzing, Phys. B: Cond. Matt. 256-258, 182 (1998).
2 A. Rosch, J. Paaske, J. Kroha, and P. Wölfle, Phys. Rev. Lett. 90, 076804 (2003).

3 A. Rosch, J. Paaske, J. Kroha, and P. Wölfle, J. Phys. Soc. Jpn. 74, 118 (2005).

${ }^{4}$ H. Schoeller, Eur. Phys. J. Special Topics 168, 179 (2009).

${ }^{5}$ H. Schoeller and F. Reinighaus, Phys. Rev. B 80, 045117 (2009).

6 S. Kehrein, Phys. Rev. Lett. 95, 056602 (2005). 
7 P. Fritsch and S. Kehrein, Ann. Phys. 324, 1105 (2009).

8 P. Fritsch and S. Kehrein, Phys. Rev. B 81, 035113 (2010).

${ }^{9}$ N. J. Craig, J. M. Taylor, E. A. Lester, C. M. Marcus, M. P. Hanson, and A. C. Gossard, Science 304, 565 (2004).

10 S. Sasaki et al., Phys. Rev. B 73, 161303(R) (2006).

11 C. Jayaprakash, H. R. Krishnamurthy, and J. W. Wilkins, PRL 47, 737 (1981); B.A. Jones and C. M. Varma, PRL 58, 843 (1987); R. M. Fye and J. E. Hirsch, PRB 40, 4780 (1989); I. Affleck, A. W. W. Ludwig, and B. A. Jones, PRB 52, 9528 (1995).

12 A. Kaminski, Y. V. Nazarov, and L. I. Glazman, Phys. Rev. Lett. 83, 384 (1999).

13 J. Paaske, A. Rosch, J. Kroha and P. Wölfle, Phys. Rev. B 70, 155301 (2004).

${ }^{14}$ V. Koerting, P. Wölfle, J. Paaske, Phys. Rev. Lett. 99, 036807 (2007).

15 V. Koerting, Ph.D. thesis, Karlsruhe (2007).

16 H. B. Heersche, Z. de Groot, J. A. Folk, L. P. Kouwenhoven, H. S. J. van der Zant, A. A. Houck, J. Labaziewicz, and I. L. Chuang, Phys. Rev. Lett. 96, 017205 (2006).
17 S. Kehrein, The Flow Equation Approach to Many-Particle Systems, Springer Tracts in Modern Physics, SpringerVerlag, 2006.

18 M. A. Ruderman and C. Kittel, Phys. Rev. 96, 99 (1954); T. Kasuya, Prog. Theor. Phys. 16, 45 (1956); K. Yosida, Phys. Rev. 106, 893 (1957).

19 S. Sachdev, and R. N. Bhatt, PRB 41, 9323 (1990).

20 A. A. Abrikosov, Physics 2, 5 (1965).

21 F. Wegner, Ann. Physik (Leipzig) 506, 77 (1994).

22 M. Garst, S. Kehrein, T. Pruschke, A. Rosch, and M. Vojta, Phys. Rev. B 69, 214413 (2004).

23 P. W. Anderson, J. Phys. C 3, 2436 (1966).

24 T. Korb, F. Reininghaus, H. Schoeller, and J. König, PRB 76, 165316 (2007).

25 M. Pletyukhov, D. Schuricht, and H. Schoeller, Phys. Rev. Lett. 104, 106801 (2010) .

26 H. Schmidt, P. Wölfle, Ann. Phys. 19, 60-74 (2009).

27 P. Fritsch, V. Koerting, S. Kehrein, to be published. 